Philosophie ANTIQUE

\section{Philosophie antique}

Problèmes, Renaissances, Usages

$18 \mid 2018$

L'athéisme antique

\title{
L'aporie de Protagoras sur les dieux
}

Le Peri theon et sa tradition

\section{Michele Corradi}

\section{OpenEdition}

\section{Journals}

Édition électronique

URL : https://journals.openedition.org/philosant/1016

DOI : 10.4000/philosant.1016

ISSN : 2648-2789

Éditeur

Éditions Vrin

\section{Édition imprimée}

Date de publication : 1 novembre 2018

Pagination : 71-103

ISBN : 978-2-7574-2372-1

ISSN : $1634-4561$

\section{Référence électronique}

Michele Corradi, "L'aporie de Protagoras sur les dieux », Philosophie antique [En ligne], 18 | 2018, mis en ligne le 01 novembre 2019, consulté le 03 décembre 2022. URL : http://journals.openedition.org/ philosant/1016 ; DOI : https://doi.org/10.4000/philosant.1016

\section{(c) (i) (3)}

Creative Commons - Attribution - Pas d'Utilisation Commerciale - Pas de Modification 4.0 International - CC BY-NC-ND 4.0

https://creativecommons.org/licenses/by-nc-nd/4.0/ 


\section{L'APORIE DE PROTAGORAS SUR LES DIEUX* \\ Le Peri theon et sa tradition \\ Michele Corradi \\ Aix-Marseille Université, IHP \\ michele.corradi@univ-amu.fr}

RÉsumÉ. Le célèbre incipit du Peri theon (80 B $4 \mathrm{DK}=31$ D 10 Laks-Most), dans lequel Protagoras affirmait être dans l'incapacité de savoir si les dieux existent ou non, joue sans aucun doute un rôle important dans l'histoire de l'athéisme ancien dans la mesure où il permet, comme le souligne David Sedley, de reconstruire un contexte culturel dans lequel la négation de l'existence des dieux était considérée comme une thèse philosophique digne d'être discutée. La présente contribution portera sur les principaux problèmes exégétiques relatifs au fragment protagoréen. Dans un premier moment, nous nous occuperons de la question de la constitution du texte. Nous considérerons ensuite l'historicité de la tradition biographique concernant la persécution judiciaire du sophiste, qui est évoquée par la plupart des sources citant le fragment. Finalement, nous essayerons d'étudier le fragment de Protagoras dans le cadre de la tradition philosophique et littéraire qui le précède et de l'analyser à la lumière de la réflexion philosophique du sophiste lui-même, afin de mieux comprendre le point de vue sur les dieux et la religion que Protagoras exprimait dans son écrit.

Summary. The famous incipit of Protagoras' Peri theon $(80$ B 4 DK $=31 \mathrm{D} 10$ LaksMost), where the sophist affirms that he is unable to know if the gods exist or not, plays undoubtedly an important part in the history of ancient atheism. Indeed, as D. Sedley points out, it suggests a cultural context in which atheism was an established position, available to endorse or reject. The present paper will deal with the main problems concerning Protagoras'fragment and its tradition. First, we will consider some questions related to the constitutio textus and we will then go on to analyze the biographical

* Je tiens à remercier tout particulièrement Rossella Saetta Cottone qui m'a invité à présenter une première version de ce travail lors de la séance du 18 mars 2017 du séminaire «Présocratiques » organisé au Centre Léon Robin. Mes remerciements vont aussi à Mathilde Brémond, Jean-Baptiste Gourinat, Emmanuelle Jouët-Pastré, Gérard Journée, Silvio Marino, Jean-Claude Picot et Alonso Tordesillas pour les remarques, les observations et les suggestions très stimulantes qu'ils m'ont proposées à cette occasion.

Philosophie antique, $\mathrm{n}^{\circ} 18$ (2018), 71-103 
tradition concerning Protagoras' persecution, quoted from most sources of this fragment. In conclusion, we will try to read the text within the context of the preceding philosophical and literary tradition and to analyze it in the light of other Protagorean texts, in order to understand the point of view of the sophist on gods and religion. 
Pour qui désire reconstruire l'histoire de l'athéisme ancien ${ }^{1}$, il est presque impossible de négliger le rôle que joue le célèbre fragment constituant l'incipit du Peri theon (80 B 4 DK = 31 D 10 Laks-Most 2016a et b ${ }^{2}$ ), dans lequel Protagoras affirmait de manière probablement assez scandaleuse pour son époque qu'il était incapable de savoir si les dieux existent ou s'ils n'existent pas $^{3}$.

Si la position de Protagoras apparaît, aux yeux des modernes, comme relevant de l'agnosticisme plutôt que de l'athéisme, plusieurs témoignages anciens inscrivent en revanche le nom du sophiste dans des listes de philosophes athées ${ }^{4}$. Certes, le terme grec atheos présente une gamme de sens beaucoup plus ample que le français « athée $»^{5}$. Toutefois, au moins une source ancienne, l'épicurien Diogène d'CEnoanda (fr. 16, col. II 1-III 14 Smith $=80$ A $23 \mathrm{DK}=31 \mathrm{R} 24 \mathrm{LM}$ ), cherche à démontrer, en proposant une interpré-

1. Une version plus développée de cet article est parue en langue italienne (Corradi 2017)

2. Dorénavant LM.

3. Cf. par exemple Fahr 1969 p. 92-99, Bremmer 2007 p. 12-13, Sedley 2013 p. 141, Whitmarsh 2015 p. 87-91, Winiarczyck 2016 p. 73.

4. Cf. Winiarczyck 1976 et Winiarczyck 1984 p. 177-178. Diogène d'OEnoanda (fr. 16 Smith, col II 1, III $14=80$ A 23 DK $=31$ R 24 LM), Eusèbe $(P E ~ 14,3,6-7=80$ B 4 DK = 31 R 29 LM et 14, 19, 10), Épiphane (Haer. III, p. 506, 18), Jean Chrysostome (Hom. in 1Cor. 4, 5), Augustin (Contra litteras Petiliani, III, 21, 25, où il faut toutefois peutêtre corriger le Protagoras des manuscrits en Diagoras) considèrent Protagoras comme un athée. En revanche, plusieurs sources anciennes soulignent la spécificité du point de vue de Protagoras, $c f$. par exemple Cicéron (ND 1,2) et Galien (Propr. Placit. 2, p. 172, 35-173, 2 Boudon-Millot \& Pietrobelli 2005). Philodème (Piet. PHerc. 1428, col. XIV 32-XV 8 Henrichs $1974=80$ A 23 DK) classe la position de ceux qui ne savent si les dieux existent parmi les trois formes possibles d'athéisme. Cf. Obbink 1996 p. 1-2. Sextus Empiricus (M 9, 55-57 = 80 A $12 \mathrm{DK}=31 \mathrm{R} 19 \mathrm{LM}$ ) témoigne d'une double tradition exégétique concernant le sophiste.

5. Au $v^{\mathrm{c}}$ siècle, les significations du terme les plus répandues sont « irréligieux », « immoral », « scélérat ». Le sens « abandonné des dieux » est plus rare. La première attestation dans laquelle le mot a la valeur d' « athée », « qui nie l'existence des dieux » se trouve dans l'Apologie de Socrate de Platon (26c). Cf. Winiarczyck 2016 p. 61-62. 
tation probablement forcée du texte protagoréen, que la position apparemment agnostique du sophiste implique en réalité la négation de l'existence des dieux ${ }^{6}$. Quoi qu'il en soit, comme l'a souligné David Sedley ${ }^{7}$, même s'il n'est pas possible de considérer le sophiste comme « a positive atheist », ses mots jouent un rôle fondamental dans l'histoire de l'athéisme ancien, dans la mesure où ils permettent de reconstruire un contexte culturel dans lequel la négation de l'existence des dieux était considérée comme une thèse philosophique digne d'être discutée et à la fois accueillie ou réfutée.

Le point de vue de Protagoras semble d'ailleurs avoir constitué le point de départ d'une tradition sceptique à l'égard de la religion. Ce n'est pas par hasard si un autre sophiste, Prodicos, que la tradition considère comme l'élève de Protagoras (84 A 1 DK $=34$ P 1 LM), semble avoir développé une doctrine sur les origines de la religion selon laquelle les dieux de la tradition ne seraient que la divinisation de ce qui est utile aux êtres humains (84 B 5 $\mathrm{DK}=34 \mathrm{D} 15-18 \mathrm{LM})^{8}$. Théodore l'athée lui-même semble s'être inspiré de Protagoras en choisissant d'intituler Peri theon le livre dans lequel il rejetait complètement les croyances en les dieux (Diogène Laërce II, $97=$ SSR IV H 13) 9 .

Au-delà de la question de l'attribution à Protagoras de l'étiquette d'athée, et afin de mieux comprendre la position du sophiste à propos de l'existence des dieux, il est à mon avis fondamental de revenir une fois encore sur le texte du fragment protagoréen et sur les problèmes qui concernent sa tradition pour analyser à nouveau les principales questions exégétiques le concernant qui demeurent encore ouvertes. Si l'on considère le grand nombre d'études relatives à l'autre fragment célèbre de Protagoras conservé, l'incipit de l'Aletheia (80 B $1 \mathrm{DK}=31 \mathrm{D} 9 \mathrm{LM}$ ), il est frappant de remarquer qu' il n'y a, en revanche, qu'un peu plus d'une vingtaine de contributions spécifiquement consacrées au Peri theon ${ }^{10}$, parmi lesquelles émergent les travaux de Carl Werner Müller, Jaap Mansfeld, Olof Gigon, Vincenzo Di Benedetto, Jonathan Barnes ${ }^{11}$. Si la fortune critique inégale des deux fragments peut s'expliquer par le rôle que le principe de l'homme-mesure joue dans la

6. Cf. Barnes 2015 et les remarques de M. F. Smith 2003 p. 72-73.

7. Sedley 2013 p. 346-347.

8. Comme le montre l'utilisation du verbe eíó́val, il est possible de saisir un rapport textuel entre l'incipit du Peri theon de Protagoras (80 B 4 DK) et un passage de Philodème, Piet. PHerc. 1428 fr. 19 Henrichs 1976, qui est absent du recueil de Diels et Kranz même s'il concerne probablement le scepticisme de Prodicos à l'égard de la possibilité de connaître les dieux. Cf. Mayhew 2011 p. 183-185.

9. Whitmarsh 2015 p. 123.

10. Pour s'en rendre compte, il suffit de considérer les trois cent cinquante entrées ayant comme sujet « Protagoras Abderita » qui se trouvent aujourd'hui dans l'Année philologique en ligne (http://www.annee-philologique.com).

11. Müller 1976, Mansfeld 1981, Gigon 1985, Di Benedetto 2007a, Barnes 2015. 
tradition philosophique postérieure, par exemple dans le Théétète de Platon, il est également possible d'expliquer la moindre considération de l'incipit du Peri theon par les problèmes exégétiques complexes qu'il comporte. Du point de vue de la constitution du texte, si, pour ce qui est du principe de l'hommemesure, elle ne présente pour ainsi dire aucun problème, en revanche, pour ce qui concerne le fragment sur les dieux, il n'est pas aisé de reconstruire la formulation authentique en raison du fait que la tradition offre des éléments difficiles à harmoniser. La version canonique que propose Hermann Diels ${ }^{12}$ n'est attestée en tant que telle dans aucune source ancienne, et n'est que le fruit d'une recomposition que le philologue allemand a opérée en s'appuyant sur divers témoignages. En outre, la plupart des sources qui le citent rattachent le fragment à une tradition biographique douteuse selon laquelle, suite à la lecture du Peri theon à Athènes, le sophiste aurait subi une persécution judiciaire qui l'aurait contraint à quitter la cité. Pendant le voyage, le bateau qui le transportait aurait fait naufrage et causé sa mort. Cette tradition révèle en effet plusieurs inconséquences et se heurte surtout au silence de Platon à son égard. En revanche, dans le Ménon (91e = 80 A 8 DK = 31 P 14 LM), Socrate dit explicitement que Protagoras aurait vécu une vie pleine de succès jusqu'à sa mort. Un autre problème concerne la difficulté de reconstruire les argumentations qui pouvaient faire suite à un incipit si choquant. En raison de ces difficultés, selon Kurt von Fritz ${ }^{13}$, l'existence elle-même de l'écrit Peri theon serait à remettre en question : quel genre d'écrit aurait pu se développer après un commencement si péremptoire et tranchant ?

Tous ces problèmes exégétiques complexes feront l'objet de notre contribution. Dans un premier moment, nous nous occuperons de la question de la constitution du texte. Nous considérerons ensuite la tradition biographique évoquée par les sources qui citent le fragment pour en vérifier l'historicité. Finalement, nous essayerons de placer le fragment de Protagoras dans le cadre de la tradition philosophique et littéraire qui le précède et de l'analyser à la lumière de la réflexion philosophique du sophiste lui-même afin de mieux comprendre le point de vue sur les dieux que le sophiste exprimait dans son écrit.

Nous n'avons pas la possibilité dans le cadre limité de cet article de traiter des rapports entre le Peri theon et le célèbre mythe de Prométhée que Platon attribue à Protagoras dans le dialogue éponyme $(320 \mathrm{c}-322 \mathrm{~d}=80 \mathrm{C} 1 \mathrm{DK}$ $=31 \mathrm{D} 40 \mathrm{LM})^{14}$ dans lequel les dieux jouent un rôle important, voire

12. Diels \& Kranz 1951-1952 t. 2 p. 265.

13. Fritz 1957 col. 920.

14. Le problème de l'attribution à Protagoras de l'ensemble du mythe est controversé, même si la plupart des commentateurs tendent à saisir dans le texte la présence d'un noyau authentiquement protagoréen, probablement remontant au Peri tes en archei katastaseos (Sur l'état primitif de l'homme) (80B 8b DK = $31 \mathrm{D} 1 \mathrm{LM})$, ou, selon l'hypothèse de Manuwald 
décisif, dans le développement de la civilisation humaine. Nous nous contenterons de souligner ici que la présence des dieux dans le récit de Protagoras ne doit pas être, à notre avis, considérée comme étonnante, surtout si l'on garde présent à l'esprit que le récit du sophiste a la nature d'un muthos. Le choix du muthos à la place du logos comme forme du discours que Protagoras lui-même formule avant de commencer son discours, à savoir d'une narration avec des personnages et d'une action qui se déroule dans le temps (320c $=31$ D 39 LM), implique un certain degré de fiction ${ }^{15}$. Étant donné le renvoi à la préhistoire du genre humain ${ }^{16}$ et le rapport avec la tradition poétique la concernant, Protagoras aurait pu difficilement renoncer à attribuer un rôle aux dieux ${ }^{17}$. Comme le rappelle Luc Brisson ${ }^{18}$, le but de ce mythe n'est pas de démontrer l'existence des dieux mais la participation de toute la communauté humaine à aidos et dike et de démontrer par là que l'art politique, fondé sur ces vertus, peut et doit être enseigné : compte tenu de ce que Protagoras veut démontrer, la question de l'existence des dieux n'est pas significative ${ }^{19}$.

Commençons donc notre analyse par les problèmes d'ordre textuel.

\section{Le texte}

La référence la plus ancienne au texte du fragment dont nous soyons certains se trouve dans le Théétète de Platon (162d = 80 A 23 DK), qui y fait allusion, sans pour autant le citer littéralement dans le cadre de la critique du principe de l'homme-mesure. Des citations textuelles ne se retrouvent que chez des auteurs plus tardifs. Comme on le sait, le texte proposé par Diels intègre le texte conservé par Diogène Laërce (IX, 51) avec celui d'Eusèbe $(P E 14,3,6-7=80$ B 4 DK = 31 R 29 LM et 14, 19, 10). Chez Diogène Laërce, on lit :

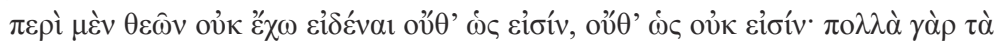

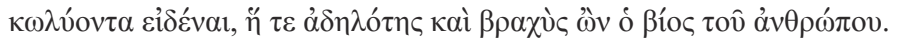

1999 p. 175, au Peripoliteias (Sur la constitution) (80B 8a DK = 31 D 1 LM). Cf. Corradi 2016 p. 337-342 pour une discussion plus ample du problème.

15. Pour les caractéristiques des mythes dans les dialogues de Platon, $C f$. au moins Most 2012.

16. $C f$. Bonazzi 2012.

17. Selon Beresford 2013 p. 139-148, l'utilisation du mythe constituerait un voile visant à cacher le caractère rationaliste, voire impie, de la doctrine.

18. Brisson 1975 p. 8-9 n. 3.

19. À ce propos, il est possible de rappeler le rapport entre muthos et logos que Platon évoque au début du Phédon (60b-c) : la transformation du logos de Socrate en muthos implique la personnification des entités en cause et le développement de l'action dans un passé indéterminé. Ce n'est pas par hasard que dans le muthos ésopique du Phédon, le dieu joue un rôle fondamental. Cf. Betegh 2009 p. 78-80. 
Quant aux dieux, je ne suis en mesure de savoir ni qu'ils existent ni qu'ils n'existent pas, car trop de facteurs nous empêchent de le savoir : l'adelotes [des dieux / de la question ?] et la brièveté de la vie humaine.

Dans ce texte, Protagoras déclare qu'il est incapable de savoir si les dieux existent ou non, incapacité qu'il justifie par la présence de nombreux obstacles. Parmi ces obstacles, Protagoras se limite à citer l'adelotes (nous reviendrons par la suite sur l'interprétation de ce mot qui signifie, en gros, « obscurité » et qui peut se référer à la fois aux dieux ou à la question même de leur existence) et la brièveté de la vie humaine. Diels insère après oủ $\theta$ ' $\dot{\omega}$ oủ c cioív

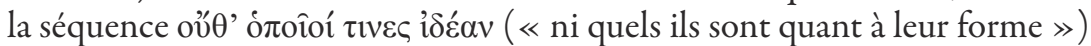
qui se retrouve dans les deux passages d'Eusèbe $(P E 14,3,6-7 \text { et 14, 19, 10 })^{20}$. Selon Protagoras, l'impossibilité cognitive ne concernerait pas seulement le problème de l'existence des dieux mais aussi celui de la détermination de leur forme. Dans l'apparat critique, Diels justifie son choix en le mettant en parallèle avec un passage d'Hérodote $(2,53)$, sur lequel nous reviendrons par la suite. Si la référence à la forme, à l'idea de la divinité n'apparaît que dans les témoignages d'Eusèbe (PE 14, 3, 7 et 14, 19, 10), Épiphane (Anc. 104, 2) et Théodoret (Affect. 2, 113), plusieurs textes confirment que le scepticisme de Protagoras concernait la possibilité de déterminer la nature, la qualité des dieux : Timon de Phlionte (fr. 5 Di Marco 1989), Cicéron (ND 1, 29 = 80 A 23 DK), Sextus Empiricus (M. 9, 56), Philodème (Piet. PHerc. 1428, col. XV 1-4 Henrichs $1974=80$ A 23 DK), Théophile (Autol. 3, 7), Valère Maxime (1, 1 ext. 7$)$. Une série de témoignages indiquent de manière variée les raisons du scepticisme de Protagoras. Certains se contentent de souligner qu'il existe plusieurs facteurs qui empêchent de parvenir à un savoir sur les dieux, notamment, pour reprendre l'heureuse formulation de Mansfeld, des « epistemological obstacles ${ }^{21}$. Une seule source, Diogène Laërce IX, 51, mentionne les obstacles, à savoir l'adelotes et la brièveté de la vie humaine. Il est en tout cas fortement probable qu'elle conserve une partie du texte que les autres sources ne citent pas $^{22}$. Il existe également une série de variantes moins importantes liées à la nature plus ou moins directe de la citation ${ }^{23}$. En premier lieu, pour exprimer l'impossibilité de parvenir au savoir à propos des dieux,

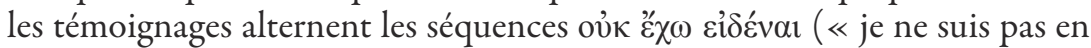

20. La séquence d'Eusèbe est reprise avec des petites variations par Épiphane Anc. 104, 2, qui attribue la phrase protagoréenne à un poète comique inconnu, un certain Eudaimon - le texte, qui est à mettre en rapport avec Théophile (Autol. 3,7 ), se base cependant sur une conjecture de Wilamowitz-Moellendorff 1911 p. 763 - et par Théodoret, Affect. 2, 113, texte qui dérive très probablement d'Eusèbe. Cf. Scholten 2015 p. 103-122 et 252-253.

21. Mansfeld 1981 p. 38-39. Outre Diogène Laërce IX, 51 et Eusèbe PE 14, 3, 7 et 14, 19, 10, font allusion à l'existence de ces facteurs Sextus M. 9, 56, Théophile Autol. 3, 7 et Épiphane Anc. 104, 2.

22. Il ne s'agit donc pas d'une interpolation. Cf. Barnes 2015 p. 201-202.

23. Un schéma très utile se trouve chez Dietz 1976 p. 132-137. 


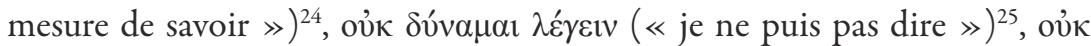
oî $\delta \alpha$ (« je ne sais pas ») $)^{26}$. La formulation de l'option concernant l'existence ou la non-existence des dieux se présente à la fois sous forme d'interrogation indirecte simple ${ }^{27}$ ou double ${ }^{28}$ ou de subordonnée complément d'objet direct $^{29}$. Si la plupart des interprètes semblent être d'accord sur le choix d'oơ

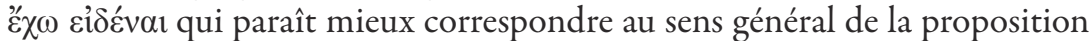
en exprimant la condition d'incapacité cognitive dans laquelle Protagoras se trouve à l'égard du problème de l'existence des dieux ${ }^{30}$, dans le cas du choix entre la subordonnée complément d'objet direct et l'interrogation indirecte, il est préférable d'attribuer à Protagoras les deux subordonnées complément d'objet direct introduites par $\hat{c}$. On pourrait à ce propos rappeler l'analogie avec le double $\hat{\omega} \varsigma$ du principe de l'homme-mesure (80 B $1 \mathrm{DK}=31 \mathrm{D} 9 \mathrm{LM})$ et souligner que le témoignage le plus ancien, celui de Platon dans le Théétète,

24. Diogène Laërce IX, 51, Suda ( $\pi 2958=80$ A 3 DK) et Scholia in Platonem (R. 600c $=80$ A 3 DK).

25. Sextus M. 9, 56, Théophile Autol. 3, 7, Épiphane Anc. 104, 2. Il faut également consi-

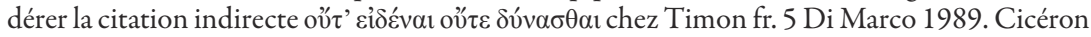
( ND 1, 63 = $80 \mathrm{~A} 23 \mathrm{DK}$ ) propose neque ... habeo dicere (Cf. Cic. ND 1, 29 où on lit la périphrase sese negat omnino de deis habere, quod liqueat).

26. Eusèbe PE 14, 3, 7 et 14, 19, 10, Théodoret Affect. 2, 113, Chrysostome Hom.in 1Cor.

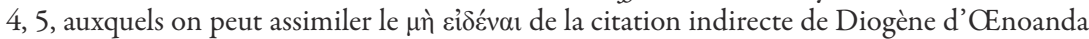

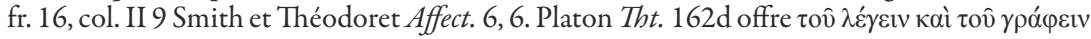

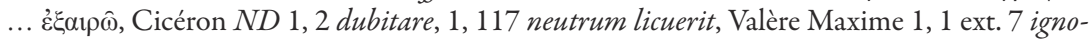
rare, Maxime de Tyr $(11,5)$ árvosiv, Galien Propr.Placit. 2, p. 172, 36 Boudon-Millot \&

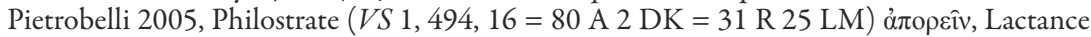
(Ira, 9, 1) non liquere.

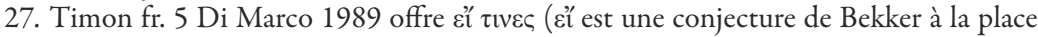

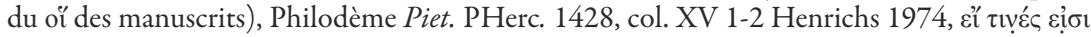

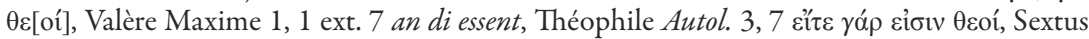

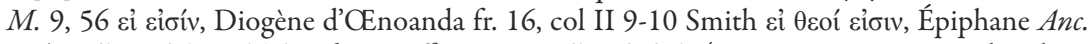

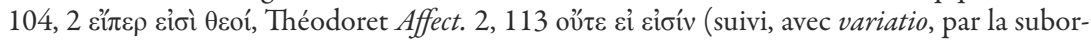

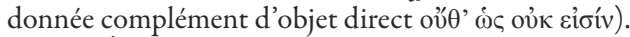

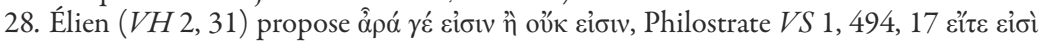

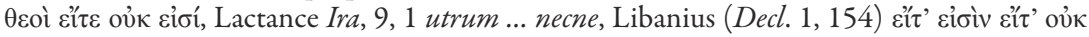

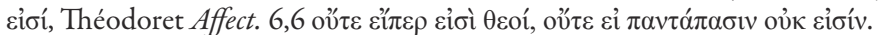

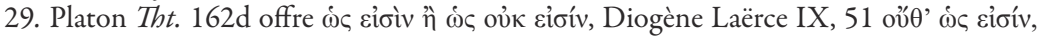
oư $\theta^{\prime}$ '

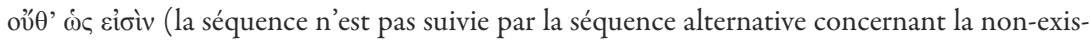

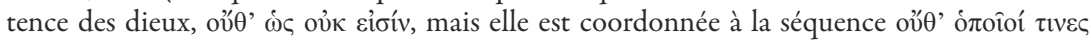

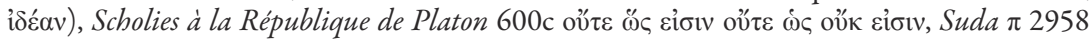

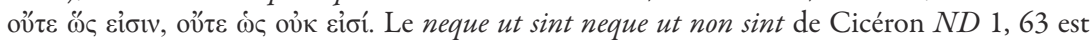
probablement un calque du grec oü $\theta$ ' construction asyndétique sint, non sint qualesve sint, 1, 117 l'infinitive double nec esse deos nec non esse). Cf. Dyck 2003 p. 144.

30. Cf. également l'habeo de Cicéron $N D$ 1, 63. Toutefois, la présence de dicere fait plutôt

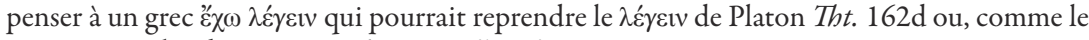

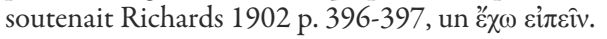


présente également le double $\mathfrak{\omega} \varsigma^{31}$.

Comme l'a mis en évidence Di Benedetto ${ }^{32}$, pour la constitution du texte, il faudrait considérer plus attentivement le $\mu \varepsilon$, sujet d'síśc $\alpha$ r qui se trouve chez Eusèbe $(P E 14,19,10)$ et qui semble être confirmé par le $\mu \varepsilon$ isolé de Sextus Empiricus (M. 9, 56), Théophile (Autol. 3, 7) et Epiphane (Anc. 104, 2 : le verbe cỉó́val doit probablement être sous-entendu). Selon le savant italien,

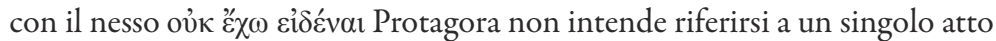
conoscitivo, $\mathrm{ma}<\mathrm{a}>$ un dato per così dire strutturale, una qualità o una capacità che contrassegni il soggetto. Il rapporto con un soggetto appare come

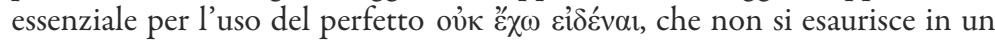
singolo atto conoscitivo.

Dans le texte reconstruit par Diels, le deuxième eíf́́vaı, que Wilamowitz ${ }^{33}$ proposait de supprimer, serait dépourvu de tout rapport avec un sujet auquel attribuer un état « contrassegnato dal "conoscere" ». L'insertion du $\mu \varepsilon$ comme

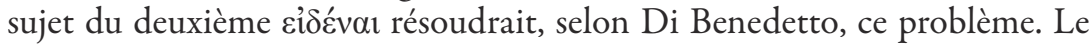
fait que le texte d'Eusèbe et celui de Sextus appartiennent à deux traditions différentes, la première concernant des questions gnoséologiques, la deuxième concernant le problème de l'athéisme, garantirait leur indépendance et renforcerait la valeur de $\mu \varepsilon$ pour la constitution du texte. Deux allusions possibles de Platon au fragment protagoréen pourraient encore renforcer cette hypothèse. Di Benedetto, dans le sillage de Maria Michela Sassi ${ }^{34}$, signale

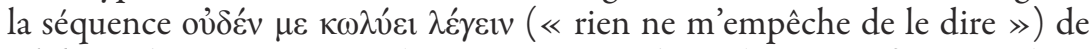
Phd. 108d-e, passage qui, selon Sassi, contiendrait plusieurs références à l'incipit du Peri theon. On pourrait encore ajouter l'allusion que Mansfeld ${ }^{35}$ croit découvrir dans le Cratyle : en se moquant de l'élève de Protagoras, Prodicos (384b5-6 = 84 A 11 DK = 34 P 5 LM), Socrate dit que, si ses moyens lui avaient permis de suivre les leçons à cinquante drachmes que le sophiste donnait sur la question, rien n'empêcherait Hermogène de savoir sur l'heure

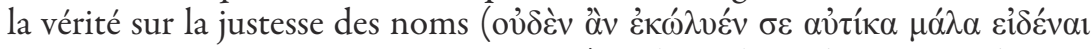

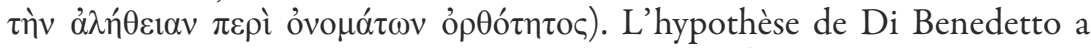
été favorablement accueillie par Tiziano Dorandi ${ }^{36}$ dans l'apparat critique de son édition de Diogène Laërce et par André Laks et Glenn Most dans leur édition des fragments et des témoignages des premiers penseurs grecs ${ }^{37}$.

31. L'interprétation de Kerferd 1981 p. 235-238 n'est pas convaincante. Kerferd attribue en effet aux $\dot{\omega} \varsigma$ du Peri theon la valeur de « comment ». Selon cette interprétation la séquence

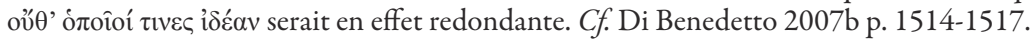

32. Di Benedetto 2007a.

33. Wilamowitz-Moellendorff 1911 p. 763.

34. Sassi 1987.

35. Mansfeld 1981 p. 42.

36. Dorandi 2013 p. 694.

37. Laks-Most 2016b p. 1244. 
Cependant, même pour ce qui est de la reconstruction de Di Benedetto, il faut souligner qu'il s'agit d'un texte composite : aucune source ne transmet

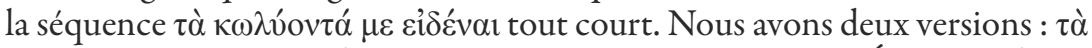

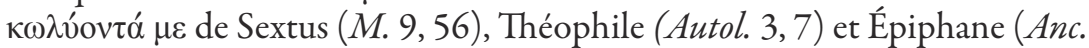

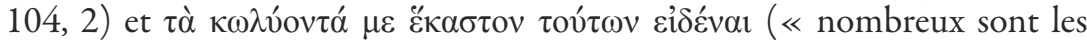
empêchements à ce que je connaisse chacun d'eux ») d'Eusèbe $(P E 14,19$, 10). Le texte sans $\mu \varepsilon$ est de toute façon acceptable d'un point de vue à la fois grammatical ${ }^{38}$ et philosophique : Protagoras justifierait sa propre incapacité personnelle de parvenir au savoir à propos de l'existence et de l'aspect des dieux par des obstacles qui concernent tous les hommes.

Les problèmes que je viens de mettre en évidence montrent, en tout cas, combien il est difficile de parvenir à une reconstitution sûre du texte originaire, ce que remarquait déjà Barnes ${ }^{39}$. Je me demande si le choix le plus correct ne serait pas celui de Mauro Bonazzi ${ }^{40}$, qui, dans sa traduction commentée des textes de Protagoras, juxtapose les diverses versions sans proposer aucune synthèse.

\section{La tradition sur le procès}

Pour ce qui est du deuxième problème évoqué concernant l'incipit du Peri theon, à savoir son rapport avec la tradition relative à la persécution judiciaire que Protagoras aurait dû subir à la suite de la lecture publique de ce texte, je me permets de rappeler des données que j'avais déjà mises en évidence dans un article de 2007, et que j'ai reprises dans ma monographie de $2012^{41}$. Le récit qui ressort des sources est plutôt intriqué et à maints égards contradictoire. La présentation des événements la plus complète se trouve chez Diogène Laërce (IX, 52-54 = 80 A 1 DK). D'autres sources font allusion tantôt à l'un, tantôt à l'autre des éléments biographiques rappelés par Diogène Laërce. Selon ce dernier (IX, 52 = 31 P 8 LM), Protagoras fut,

38. Le verbe $\kappa \omega \lambda v ́ \omega$ avec eỉó́val dans une construction impersonnelle se trouve par

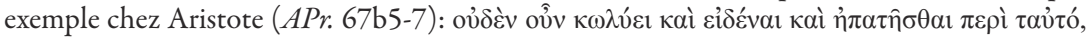

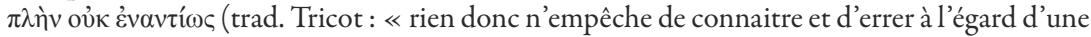
même chose, pourvu que cette connaissance et cette erreur ne soient pas contraires »). La construction de $\kappa \omega \lambda v ́ \omega$ au participe, suivi par une infinitive sans sujet exprimé se trouve par

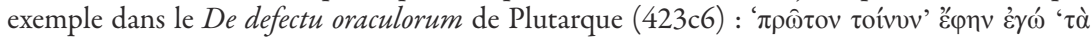

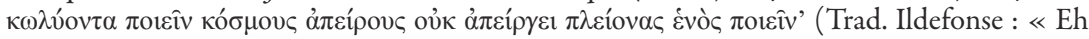
bien, tout d'abord, dis-je, ce qui empêche d'admettre des mondes en nombre illimité n'exclut

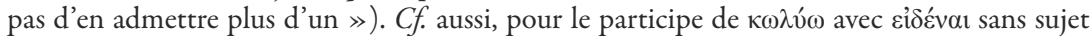

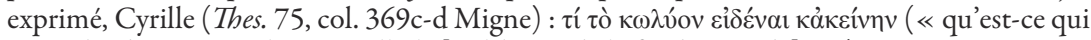
empêche de savoir également celle-là [sc. l'heure de la fin du monde] ? »).

39. Barnes 2015 p. 200.

40. Bonazzi 2009 p. 76-79.

41. Corradi 2007 et Corradi 2012b p. 31-43. 
à cause de l'incipit de son livre, expulséf2 par les Athéniens, qui brûlèrent ses livres sur la place publique, après les avoir rassemblés par voie de héraut auprès de tous ceux qui les possédaient ${ }^{43}$.

Peu après, Diogène Laërce précise que le premier des ouvrages que Protagoras lut en public fut le traité Sur les dieux ${ }^{44}$. La lecture du logos se tint à Athènes. Diogène Laërce rappelle à ce propos trois traditions différentes : la lecture aurait eu lieu dans la maison d'Euripide ou, selon certains, chez Mégaclide ; d'autres disent au Lycée (dans ce cas, le disciple de Protagoras, Archagoras, fils de Théodote, aurait prêté sa voix à son maître). Le biographe met en relation ces informations avec l'histoire de la persécution de Protagoras, en proposant deux traditions concernant le nom de son accusateur : Pythodore, fils de Polyzèlos, l'un des Quatre-Cents ou, selon le témoignage d'Aristote, Euathlos (IX $54=867$ Gigon $=80$ A 1 DK $=31$ P 19-20 LM) ${ }^{45}$.

Ensuite, Diogène Laërce rapporte une tradition remontant à Philochore (328 F 217 Jacoby), probablement liée encore une fois à l'histoire de la

42. Cicéron $N D$ 1, 63 - qui est également la source de Minucius Felix $(8,3)$-, Valère Maxime 1, 1, ext. 7, Eusèbe PE 14, 19, 10 et Libanius Decl. 1, 153-154 rappellent l'expulsion de Protagoras. Timon fr. 5 Di Marco 1989, Flavius Josèphe (Ap. 2, 266), Sextus Empiricus M. 955-57, Cyrille Iul. VI 189, 76, col. 789 B Migne (si l'on accepte la conjecture de Meier 1833,

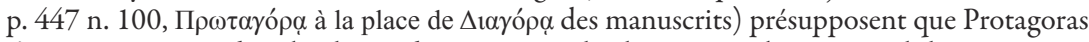
s'était sauvé avant de subir la condamnation ou le châtiment subséquent. Philostrate VS 1, 494, 21-26 rapporte deux versions : Protagoras aurait été banni après un procès ou simple-

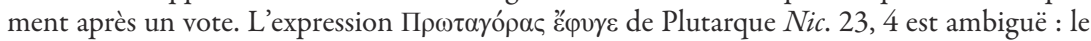
verbe peut signifier à la fois « être exilé » et «être accusé dans un procès ».

43. Le bûcher sur lequel les livres de Protagoras furent brûlés est cité par Cicéron $N D 1$, 63, avec Minucius Felix 8, 3 et Lactance Ira 9, 2, Valère Maxime 1, 1, ext. 7, Eusèbe PE 14, 19, 10 et Chron. p. 113, 21-23 Helm $=80$ A 4 DK (Cf. aussi 357, 20-22 Helm), Sch. Pl. R. 600c, Suda $\pi$ 2958. Timon fr. 5 Di Marco 1989 parle d'une volonté générique de la part des Athéniens de réduire en cendres les écrits du sophiste. La véridicité historique de l'épisode du bûcher a été remise en question par plusieurs commentateurs modernes. Hormis pour ce qui concerne le célèbre cas du bûcher où furent brûlés des textes sacrés juifs sous Antiochos IV Épiphane qui cite le Ir livre des Maccabées $(1,56)$, il s'agit d'une forme de châtiment typiquement romaine, qui serait donc peu vraisemblable pour l'Athènes de la fin du $\mathrm{V}^{\mathrm{e}}$ siècle. Cf. Wallace 2006. Piccirilli 1997 se prononce en faveur de l'historicité de l'épisode, et propose un inventaire très utile des cas où des livres furent brûlés dans le monde ancien. $C f$. aussi Dreßler 2010 p. 94-95.

44. Comme le soulignait déjà Untersteiner 1993 t. 1 p. 21-22, il n'est pas facile d'harmoniser d'un point de vue chronologique cette donnée avec l'épilogue tragique de l'histoire. Pour surmonter cette difficulté, Carbonara Naddei 1975 p. 475 propose une interprétation

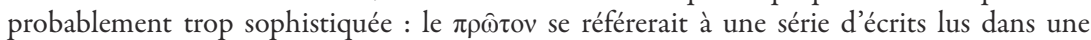
circonstance particulière. $C f$. Hourcade 2009 p. 36.

45. À partir de Derenne 1930 p. 51-54, les commentateurs n' interprètent pas l'indication de l'appartenance de Pythodore aux Quatre-Cents comme un indice pour dater le procès en 411. Le renvoi à Euathlos, au travers d'Áristote, concerne probablement un autre procès que Diogène Laërce cite par la suite (IX, $56=31 \mathrm{P} 17 \mathrm{LM}$ ), le procès à propos du paiement des honoraires qui avait opposé le sophiste à Euathlos. Cf. Corradi 2012b p. 37-43. 
persécution judiciaire du sophiste de la part des Athéniens : pendant qu'il faisait voile vers la Sicile, probablement en essayant de s'échapper d'Athènes, son navire fit naufrage. Selon l'atthidographe, Euripide ferait une allusion masquée à cet épisode dans son Ixion (DL IX 55 = T 41 Kannicht = 80 A 1 $\mathrm{DK}=31$ P 23 LM). Diogène Laërce ajoute finalement que, selon une autre tradition, Protagoras serait mort pendant le voyage, vers l'âge de quatrevingt-dix ans ${ }^{46}$.

Le panorama qui se dégage de Diogène Laërce et des autres sources a posé plusieurs problèmes aux commentateurs modernes. Il s'insère en effet dans le cadre d'un débat critique plus ample concernant la réalité historique d'une persécution qu'une série d'intellectuels fameux, surtout ceux qui avaient été les plus proches de Périclès, auraient subie, par le biais de l'accusation d'impiété, pendant les années de la guerre du Péloponnèse. Une tendance longtemps dominante, qui accueillait de façon acritique l'ensemble de la tradition sur les procès d'asebeia, bien attestée par l'étude d'Eudore Derenne ${ }^{47}$, avait conduit Eric Dodds ${ }^{48}$ à interpréter cette tradition comme la preuve de l'existence à la fin du $\mathrm{V}^{\mathrm{e}}$ siècle d'une réaction aux « Lumières » de l'époque de Périclès. Ce point de vue a été remis en question par un article fondamental de Kenneth Dover ${ }^{49}$. Dans cette étude, dont les conclusions ont été reprises et approfondies par les recherches de Robert Wallace ${ }^{50}$, le savant anglais reconsidérait l'ensemble des sources anciennes pour montrer avec des arguments plutôt persuasifs que, dans la formation de cette tradition, la fiction prévalait sur la vérité historique. À son tour, la tendance sceptique, qui a été largement acceptée après l'étude de Dover ${ }^{51}$, est aujourd'hui remise en question par les études les plus récentes. Dans les dernières années, on assiste à une réévaluation positive de la tradition d'un point de vue historique : il suffit de penser aux travaux d'Alexander Rubel, Jan Dreßler et Tim Whitmarsh ${ }^{52}$. Dans ce panorama, les recherches de Jakub Filonik constituent

46. Outre Philocore (328 F 217 Jacoby), Sextus M. 56-57, Athénée (13, 611b, si on

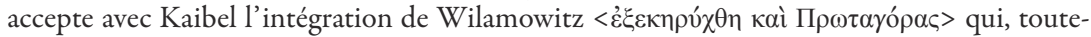
fois, n'est pas nécessaire ; $c$. Kaibel 1887-1890, vol. 3 p. 348), Philostrate $V S$ 1, 494, 24-26, Sch. Pl. R. 600c, font également allusion au naufrage. Diogène Laërce lui-même dans son épigramme consacrée à Protagoras (IX, 56) met en relation le naufrage avec la fuite. En revanche, Fritz 1957 col. 910-911 pense que la tradition sur le naufrage était originairement indépendante de celle sur la persécution. Platon ( Hp. Ma. 282d-e = 80 A 9 DK) fait référence à l'activité de Protagoras comme sophiste en Sicile.

47. Derenne 1930.

48. Dodds 1951 p. 179-204.

49. Dover 1988.

50. Par exemple Wallace 1994 et Wallace 1996.

51. Cf. par exemple Raaflaub 2000 ou Lefkowitz 2012 p. 94.

52. Rubel 2000, Dreßler 2010 p. 63-138, Dreßler 2014 p. 219-333 et Whitmarsh 2015 p. 115-124. 
une exception notable ${ }^{53}$. Une position intermédiaire et équilibrée est, en revanche, soutenue par Marek Winiarczyck ${ }^{54}$.

Malgré quelques prises de position importantes en faveur de la véridicité historique du cadre contradictoire qui émerge des sources anciennes ${ }^{55}$, je continue de penser ${ }^{56}$ que, dans le cas de Protagoras, la fiction prévaut sur la réalité historique. Dans une contribution remarquable, Carl Werner Müller ${ }^{57}$ faisait remonter l'ensemble de la tradition sur la persécution de Protagoras à Philochore, historien non exempt de partialité, et proche de l'exaltation des mœurs religieuses des Athéniens. Il semble bien que l'histoire de Protagoras suive le même schéma que celui que Philochore utilise, en se distinguant des autres sources, pour narrer la fin tragique de Phidias (328 F 121 Jacoby). De même que Phidias, coupable à l'égard de la divinité de hierosulia pour avoir volé du matériel précieux destiné à la construction de la statue d'Athéna, aurait subi un procès et aurait été contraint à la fuite mais n'aurait pas échappé au juste châtiment, tué par les Éléens après avoir sculpté la statue de Zeus à Olympie, de même, l'impie Protagoras, contraint de se sauver à l'issue du procès que les Athéniens lui avaient intenté, aurait trouvé son juste châtiment dans le naufrage, à savoir le destin proverbial qui attend les impies ${ }^{58}$. Le fondement de la reconstruction de Philochore ne serait que l'interprétation du vers de l'Ixion dans lequel Euripide aurait fait allusion au naufrage du sophiste. Après Philochore, la tradition aurait connu une double postérité, l'une dans le cadre de la doxographie parvenant, probablement à travers Clitomaque, aux listes des philosophes athées, l'autre de type biographique.

Pour solide et acceptable qu'elle soit, l'hypothèse de Müller peut être aujourd'hui remise en question, au moins partiellement. En fait, on ne peut attribuer avec certitude à Philochore que la donnée du naufrage ${ }^{59}$ : certains éléments de la tradition pourraient avoir une origine externe à l'atthidographe et pourraient être apparus plus tard, avant d'intégrer par la suite son récit $^{60}$. Le témoignage de Philostrate $(V S 1,494,8-30=80$ A $2 \mathrm{DK}=31 \mathrm{R}$

53. Par exemple Filonik 2013.

54. Par exemple Winiarczyck 2016 p. IX-X.

55. Surtout celle de Piccirilli 1997, sur lequel s'appuient les analyses de Rubel 2000 p. 64-68 et Dreßler 2010 p. 89-99, et, dans une autre perspective, celle de Capra 2000.

56. Cf. Corradi 2012b p. 31-38.

57. Müller 1976 p. 323-339.

58. Sur la tradition complexe et à maints égards contradictoire concernant la persécution judiciaire de Phidias, $c f$. au moins Raaflaub 2000 p. 101-107 et Filonik 2013 p. 26-28. Sur le topos biographique du naufrage comme châtiment divin, cf. Grau 2010 p. 371-373.

59. Cf. déjà Fritz 1957 col. 910-911.

60. Cf. Bolonyai 2007 p. 247-263. Plus particulièrement, l'histoire du bûcher des livres dériverait de Timon fr. 5 Di Marco, lequel, toutefois, comme l'avait déjà mis en évidence Dover 1988 p. 141-145, ne rapporte pas l'histoire comme s'il s'agissait d'un événement réellement arrivé : il ne s'agirait que d'un simple désir des Athéniens, jamais mis en œuvre. Cepen- 
$25 \mathrm{LM}$ ), qui met en relation la tradition sur la persécution judiciaire avec les origines de l'agnosticisme de Protagoras, apparaît comme plutôt excentrique : en s'appuyant probablement sur les Persika de Dinon ${ }^{61}$, le sophiste soutient que la position de Protagoras dériverait de l'enseignement des Mages qui, dans leurs cérémonies secrètes, invoquent la divinité mais, en public, nient son existence, pour éviter que l'on pense qu'ils tiennent leur pouvoir de la divinité. Le père de Protagoras, qui avait eu l'honneur d'accueillir dans sa maison à Abdère le roi Xerxès, aurait en effet obtenu que son fils reçût l'éducation des Mages. L'histoire, que Diels ${ }^{62}$ qualifie de « roman », semble réélaborer de façon originale diverses traditions sur Protagoras. D'une part, le lien entre la position de Protagoras et la religion perse pourrait remonter à une évaluation négative des croyances perses répandue dans la littérature grecque. D'autre part, l'opposition chez les Mages entre athéisme public et religiosité privée pourrait avoir pour modèle l'opposition entre une doctrine publique et une doctrine secrète que Platon attribue à Protagoras dans le Théétète $(152 \mathrm{c}=31 \mathrm{R} 6 \mathrm{LM})^{63}$.

Quoique l'hypothèse de Müller sur l'origine de l'ensemble de la tradition sur la persécution de Protagoras puisse être à présent nuancée, il me semble que le témoignage de Platon demeure encore un élément décisif pour nier toute vraisemblance historique à cette tradition. Dans le Protagoras (316c5$317 \mathrm{c} 5=80$ A $5 \mathrm{DK}=31 \mathrm{P} 2 \mathrm{a} \mathrm{LM}$ ), tout en mettant en évidence les risques du métier de sophiste et plus particulièrement la haine que l'on voue à ceux qui se consacrent à cette profession, Protagoras déclare qu'il exerce sa techne sans problème grâce à quelques précautions qu'il a su prendre. Le témoignage du Ménon est encore plus explicite (90e-92a = 80 A 8 DK). Dans un dialogue très tendu avec Anytos, qui se montre violemment critique à l'égard de la paideia des sophistes, Socrate remarque que, grâce à son activité d'éducateur, Protagoras a gagné des sommes bien plus importantes que Phidias, qui a pourtant produit des œuvres extraordinaires, et que dix autres sculpteurs : pendant plus de quarante ans, Protagoras aurait donc réussi à tenir caché à toute la Grèce qu'il a corrompu ceux qui le fréquentaient. Selon Socrate, Protagoras serait mort à l'âge d'environ soixante-dix ans, après avoir exercé sa profession pendant quarante ans : pendant tout ce temps, et encore jusqu'à l'époque du dialogue, sa réputation ne se serait jamais affai-

dant, selon Di Marco 1989 p. 123-124, l'utilisation de l'imparfait ह̌ $\theta \varepsilon \lambda$ ov (ils voulaient) chez Timon n'exclurait pas la réalisation effective du projet.

61. Comme le souligne Lenfant 2009 p. 120-123, la forme utilisée par Philostrate pour le nom du père de Protagoras confirmerait le rapport avec les Persika de Dinon. Cependant, on ne voit pas de manière évidente ni jusqu'à quel point Philostrate reprend Dinon ni quel rapport subsiste entre Dinon et Philochore. Cf. Müller 1976 p. 338-339.

62. Diels \& Kranz 1951-1952 t. 2 p. 255.

63. La fin tragique de Protagoras ne serait que la juste conséquence de l'enseignement de méchants maîtres. Sur le topos de l'impiété des Perses, Cf. Gazzano 2014 p. 146-152. 
blie. Nonobstant les nombreux efforts que les commentateurs ont déployés, il me semble difficile d'harmoniser la page platonicienne avec ce qui émerge des autres sources à propos de la persécution de Protagoras ${ }^{64}:$ les paroles que Platon fait prononcer à Socrate face à l'un de ses accusateurs du procès de 399 exprimeraient une ironie vraiment macabre et, à mon avis, étrangère à l'art de Platon ${ }^{65}$. En outre, les interprètes oublient souvent de considérer la suite du passage du Ménon qui est absente du recueil de Diels-Kranz (92b) : Anytos stigmatise la folie des poleis qui permettent aux sophistes de venir

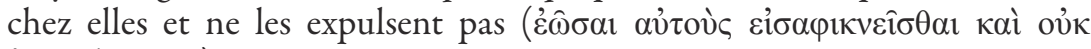

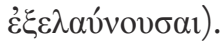

Si donc le texte de Platon s'oppose clairement à cette tradition concernant les événements qui ont suivi la lecture publique du Peri theon, il n'est pas aisé d'établir quelle était l'origine des informations sur lesquelles se fonde le récit de Philochore et des autres sources. Assurément, une tradition concernant une hostilité à l'encontre de Protagoras, indépendante de l'histoire narrée par Diogène Laërce, existe. Les passages du Protagoras et du Ménon que nous venons de citer font ressortir l'idée d'une animosité très répandue à l'égard des sophistes, dont Protagoras lui-même aurait été victime. Dans cette perspective, il faudrait rappeler un fragment des Flatteurs d'Eupolis (157 PCG), dans lequel Protagoras est qualifié d'à $\lambda \iota \tau n ́ p ı c, ~ i m p i e{ }^{66}$. Ces éléments ont

64. La remarque de Guthrie 1969 p. 269, souvent reprise par les commentateurs postérieurs, selon laquelle on pourrait harmoniser ce que Socrate dit à propos de la durable renommée de Protagoras avec la tradition sur sa persécution, car Platon aurait pu dire la même chose à propos de Socrate qui avait été condamné à mort, ne me semble s'appuyer sur aucun témoignage textuel.

65. Capra 2000 pense que Platon aurait pu opérer une distorsion de la réalité historique dans une perspective littéraire. $C f$. aussi Lenfant 2002 p. 139. L'hypothèse est intéressante mais je crois que l'on peut être d'accord avec Brancacci 2002 p. 27, selon lequel les paroles de Socrate dans le Ménon assumeraient dans ce cas le caractère d'un « crudele dileggio, non privo di una nota gratuita ». Selon Gigon 1985 p. 432-433, le témoignage platonicien pourrait de toute façon se concilier avec l'histoire du naufrage, s'il ne s'agissait que d'un malheur arrivé pendant un simple voyage en Sicile. Certains interprètes saisissent une allusion à la fin tragique de Protagoras dans une curieuse image du Théétète (171d), selon laquelle on suppose que Protagoras aurait pu hisser sa tête hors de terre jusqu'aux épaules pour réfuter les arguments avancés contre lui par Socrate et Théodore et, ensuite, s'échapper précipitamment. D'autres interprétations de l'image, probablement plus persuasives, sont toutefois possibles, cf. Corradi 2012 b p. 34 n. 2.

66. Comme cela ressort de la riche analyse de Napolitano 2012 p. 101-104, le terme, qui présente dans plusieurs passages des poètes comiques une valeur dépourvue de toute connotation politique et religieuse, peut être mis en relation, dans le fragment d'Eupolis, avec le caractère sacrilège des doctrines de Protagoras. Comme cela ressort du texte du fragment, l'impiété serait liée à une réflexion sur les meteôra, que les autres sources concernant Protagoras n'attestent toutefois pas clairement. Néanmoins Plutarque Nic. 23, 4, cite le procès subi par le sophiste dans le cadre d'une hostilité plus générale des Athéniens à l'égard des soi-disant

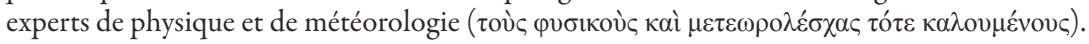


assurément joué un rôle dans la genèse de cette tradition. Cependant, il est impossible de nier la présence, dans l'ensemble de notre tradition, d'une série de traits propres à la recherche érudite de la fin du $\mathrm{IV}^{\mathrm{e}}$ siècle, surtout de ceux propres à celle issue de l'école péripatéticienne ${ }^{67}$ : l'utilisation des textes poétiques pour la reconstruction biographique, et dans ce cas spécifique, l'allusion présumée au naufrage de Protagoras dans l'Ixion d'Euripide $^{68}$; le rapport entre Euripide et les représentants à la fois les plus avancés et les plus subversifs de la culture philosophique de son époque ${ }^{69}$; le motif de l'incompréhension et du mépris des intellectuels les plus renommés de la part des Athéniens ${ }^{70}$. Il est donc possible que la recherche érudite de la fin du $\mathrm{IV}^{\mathrm{e}}$ siècle, plus particulièrement l'école péripatéticienne, ait fourni une contribution décisive à la formation de la tradition sur la persécution judiciaire de Protagoras et, plus généralement, sur les procès subis par plusieurs intellectuels à Athènes à la fin du $\mathrm{V}^{\mathrm{e}}$ siècle. Il va sans dire que l'histoire du procès de Socrate et le texte de l'Apologie de Socrate de Platon ont joué un rôle de modèle dans la formation de cette tradition ${ }^{71}$. D'ailleurs, Timon lui-même (fr. 5 Di Marco 1989) met de façon anachronique en relation l'histoire de Socrate et celle de Protagoras.

\section{Problèmes d'interprétation}

Une fois écartée la vraisemblance historique du contexte biographique relatif à la lecture publique du Peri theon, au moins pour ce qui est d'une approche d'ensemble, reste à aborder le problème de l'interprétation du texte. Par commodité, nous suivrons le texte selon la reconstruction de Diels. Comme on le sait, le fragment constituait l'incipit de l'ouvrage, comme le

67. Sur les caractères de la recherche érudite péripatéticienne, $c f$. au moins Montanari 2012. Sur la soi-disant méthode de Chaméléon, voir plus particulièrement Arrighetti 2008.

68. Pour ce qui est de la modalité d'utilisation des témoignages des poètes dans la biographie péripatéticienne, $c f$. Schorn 2012 p. 419-423.

69. Cf. Schorn 2004 p. 197-201 et Lefkowitz 2012 p. 88-90.

70. Le motif est un topos dans l'école péripatéticienne. Selon Héraclide Pontique, les accusations injustifiées à l'encontre des intellectuels les plus célèbres étaient une attitude constante des Athéniens : il avance les cas d'Homère, de Tyrtée et de Socrate (fr. 97-98 Schütrumpf). Dans l'Apologie de Socrate, Démétrios de Phalère parle du phthonos des Athéniens à l'encontre de Diogène d'Apollonie (107 SOD = Fortenbaugh \& Schütrumpf 2000) et du rapport difficile d'Héraclite (106 SOD) et de Démocrite (108 SOD) avec Athènes. Praxiphane (21 Matelli 2012) rappelle le cas de Thucydide. Cf. Corradi 2012a p. 509-511.

71. Comme le souligne Lefkowitz 1987 p. 157-161, Aristote lui-même, qui, comme nous l'avons rappelé, s'était intéressé au différend entre Protagoras et Euathlos, a joué un rôle dans la formation de traditions douteuses du point de vue historique concernant les mésaventures judiciaires de plusieurs intellectuels du $\mathrm{V}^{\mathrm{e}}$ siècle, par exemple d'Eschyle (EN 1111a8-11 $=\mathrm{T} 93 \mathrm{a}$ Radt $)$ et d'Euripide ( $R h .1416 \mathrm{a} 28-34=\mathrm{T} 98 \mathrm{Kannicht}$ ). Les remarques d'Aristote ont été reprises et amplifiées par la suite dans les recherches de péripatéticiens. Cf. Schorn 2004 p. 279-285. Sur le rôle du Socrate de Platon dans le développement du genre biographique, $c f$. Erler 2007. 


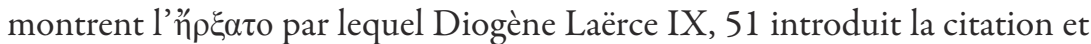

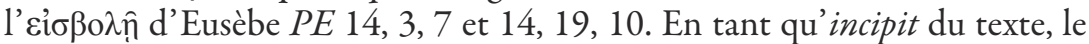
fragment était donc placé en un lieu très important dans les œuvres littéraires anciennes. Faute de pouvoir dans le cadre limité de cet article approfondir la question, nous nous limiterons donc à renvoyer à la contribution de Gian Biagio Conte ${ }^{72}$ qui est fondamentale sur ce sujet.

Le texte de Protagoras s'ouvre par une référence au sujet de l'ouvrage, Sur les dieux, dans la forme canonique introduite par $\pi \varepsilon \rho i^{73}$. Selon une modalité assez habituelle dans les incipit présocratiques, le discours se déroule à la première personne du singulier ${ }^{74}$ : Protagoras déclare qu'il est incapable de parvenir au savoir à propos de l'existence des dieux ${ }^{75}$. Le verbe être a ici en effet manifestement une valeur existentielle : il s'agirait, selon Charles Kahn, d'une des premières occurrences sûre du verbe avec cette valeur ${ }^{76}$. Il faut également remarquer la structure antithétique, marquée par l'opposi-

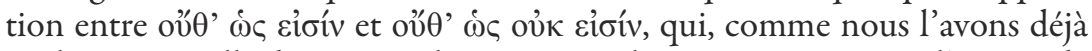
souligné, rappelle de très près la même antithèse qui caractérisait l'incipit de la Vérité, le célèbre principe de l'homme mesure (80 B $1 \mathrm{DK}=31 \mathrm{D} 9 \mathrm{LM})$ :

l'homme est la mesure de toutes les choses : de celles qui sont, du fait qu'elles

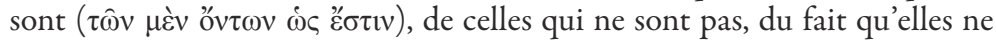

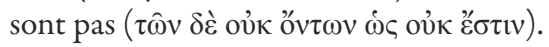

On a supposé, de façon tout à fait plausible, que cette structure antithétique anticipait la structure antilogique par laquelle l'ouvrage discutait le problème de l'existence des dieux. Selon le principe de l'antilogie, Protagoras aurait opposé un discours qui argumentait en faveur de l'existence des dieux à un discours qui la niait ${ }^{77}$. Dans l'incipit du Peri theon, le problème de l'existence des dieux se double d'un deuxième problème, étroitement lié au premier, à savoir celui de la forme des dieux. Selon une stratégie argumen-

72. Conte 2012 p. 78-117. Pour ce qui est des présocratiques, voir la remarquable contribution de Gemelli Marciano 2007.

73. Par exemple le pseudo-Xénophon (Ath. 1.1) et Hippocrate (Morb. Sacr. 1, 1, Nat. Mul. 1). Cf. aussi Alcméon 24 B 1 DK $=23$ D 4 LM.

74. Par exemple le début des Katharmoi d'Empédocle (31 B 112 DK $=22$ D 4 LM), Démocrite 68 B 165 DK = 27 P 44 e 27 D 26 LM (qui constituait probablement l'incipit du Mikros diakosmos), Hippias 86 B 6 DK $=36$ D 22 LM, Hippocrate Carn. 1. La construction

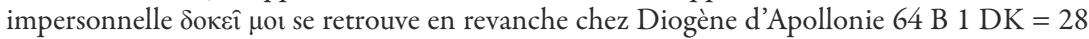
D 2 LM et dans l'incipit de la Periegesis d'Hécatée de Milet FGH 1 F 1. Cf. aussi l'insistance sur le pronom de première personne du singulier dans l'incipit du Peri phuseos de Parménide (28 B 1 DK = 19 D 4 LM). Sur le même thème, la contribution de Robbiano 2006 p. 65-70 est très stimulante.

75. Müller 1976 p. 319 n. 29 souligne à juste titre l'originalité d'une déclaration d'ignorance par rapport aux topoi de l'exorde.

76. Kahn 2003 p. 302.

77. Cf. par exemple Gigon 1985 p. $438-439$ et Tordesillas 2009 p. 16. Selon Untersteiner 1993 t. 1 p. 30-31, le logos sur les dieux était le premier des Antilogies. 
tative qui pourrait rappeler l'enchaînement des trois thèses du Peri tou me ontos de Gorgias (82 B $1 \mathrm{DK}=32 \mathrm{D} 26 \mathrm{LM})^{78}$, une fois admise hypothétiquement l'existence des dieux, Protagoras ne serait même pas en mesure de savoir quels ils sont quant à leur forme. Il est donc probable que, à la suite du problème de l'existence des dieux, l'ouvrage aurait traité celui de leur

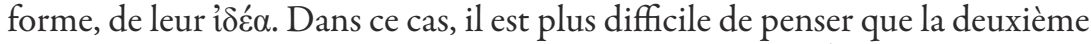
partie de l'ouvrage avait elle aussi une forme antilogique (même si l'on peut de toute façon supposer une antilogie portant sur la forme humaine ou non humaine des dieux ${ }^{79}$ ).

Néanmoins, le résultat auquel Protagoras parvenait dans la deuxième partie était également sceptique. Le scepticisme de Protagoras reposait sur la présence d'une multiplicité de facteurs qui empêchent de parvenir au savoir sur les deux questions ${ }^{80}$. Protagoras, étrangement, se limite à en citer deux : l'adelotes et la brièveté de la vie humaine. Si les commentateurs s' accordent sur l'interprétation du deuxième facteur, l'interprétation du terme adelotes est, quant à elle, débattue. La plupart des commentateurs l'interprètent de façon plus générale en considérant qu'il se réfère à la question de l'existence et de la nature des dieux, selon la signification que le terme assume dans les autres occurrences qui, excepté le Peri theon, ne sont pas antérieures au $\mathrm{II}^{\mathrm{e}}$ siècle av. J.-C. ${ }^{81}$. Voici quelques exemples : Laks et Most ${ }^{82}$ proposent l'« obscurité du sujet » et «its obscurity ${ }^{83}$, Bonazzi « obscurité du propos ${ }^{84}$ et « oscurità dell'argomento » ${ }^{85}$, Daniel W. Graham ${ }^{86}$ the « obscurity of the

78. L'analogie avec Gorgias ne serait toutefois pas pertinente selon Bodéüs 1987 p. 246-247. Sur le rapport entre Protagoras et le traité gorgien, cf. Corradi 2015 p. 448-449.

79. Des restes du nom «Protagoras » sont probablement lisibles dans PHerc. $124 \mathrm{fr}$. 2 De Falco 1923 contenant des morceaux d'un ouvrage de Démétrios Lacon dans lequel on traitait probablement le problème de l'anthropomorphisme des dieux. Cf. Romeo 1988 p. 33.

80. L'expression $\pi \mathrm{o} \lambda \lambda \dot{\alpha} \tau \grave{\alpha} \kappa \omega \lambda v ́ o v \tau \alpha$ est, selon Mansfeld 1981 p. 40 n. 6, idiomatique : chez Hérodote $(8,144,2)$ on ne cite que trois obstacles, chez Hippocrate (Vict. 3, 67) quatre.

81. Cf. Dietz 1976 p. 137. Les occurrences les plus anciennes se réfèrent à l'incertitude dans les domaines militaire (Polybe 5, 2, 3 et 36, 6, 2, Plutarque Cat.Min. 59, 11, Demetr. 15, 4, Per. 18, 1 et 28, 8, Denys d'Halicarnasse 9, 35), économique (Plutarque Apophthegmata Laconica 1, 27d, Crass. 2, 5, Saint Paul 1 Ep. Tm. 6, 17) et politique (Plutarque Caes. 7, 2, Oth. 9,2 ), à l'incertitude à propos du futur, du destin et, plus généralement, des choses humaines (Philon, Deus 29, Legat. 51 et 322, Virt. 152, Plutarque Cons. Apo. 112d, Alex. 69, 5, Cam. 32, 3, Cornutus ND 12, 14-16). Dans une signification plus philosophique, l'adelotes concerne l'au-delà chez Plutarque fr. 178 Sandbach, l'essence de l'âme chez Philon Mutat. 10, les pragmata chez Philon Quest.Gen. 2, fr. 54a. Le terme est naturellement connoté en un sens sceptique chez Sextus Empiricus $(M .8,374$; 8, 452 et 11, 229 renvoient à des problèmes logiques, M. 1,305 à l'attitude de Pyrrhon).

82. Laks \& Most 2016b p. 1244.

83. Laks \& Most 2016a p. 42.

84. Bonazzi 2009 p. 52.

85. Bonazzi 2007 p. 125.

86. Graham 2010 p. 707. 
question », Maurizio Migliori, Ilaria Ramelli et Giovanni Reale ${ }^{87}$ « oscurità della questione », José Solana Dueso ${ }^{88} \ll$ la 'oscuridad' », Thomas Schirren et Thomas Zinsmaier ${ }^{89}$ « die Undeutlichkeit der Sachlage ».

On pourrait citer en faveur de cette première interprétation une curieuse anecdote qui se trouve chez Cicéron $(N D 1,60)$, et qui semble réélaborer dans la forme de l'anecdote certains des éléments fondamentaux de la réflexion protagoréenne. Les protagonistes de l'anecdote sont le tyran Hiéron et le poète Simonide. Hiéron demanda à Simonide ce qu'est un dieu et quelle est sa nature. Le poète

sollicita un jour de réflexion; le lendemain, comme Hiéron lui répétait la question, il demanda deux jours ; quand il eut ainsi doublé le nombre des jours à plusieurs reprises, Hiéron étonné lui demanda de s'expliquer. « C'est que, dit-il, plus je réfléchis, plus l'espoir d'y voir clair s'obscurcit » (quanto diutius considero... tanto mihi res videtur obscurior) (trad. Auvray-Assayas) ${ }^{90}$.

Dans le texte, il est donc possible de remarquer une relation entre le problème de la nature des dieux (en revanche leur existence n'est pas remise en question) et la temporalité de la vie humaine, rapport qui met en évidence plusieurs analogies avec la position protagoréenne. Manifestement, le texte cicéronien s'accorde avec les interprétations du mot adelotes que nous venons de citer en considérant que l'obscurité renvoie à la question elle-même. En revanche, d'autres interprètes proposent des traductions pour ainsi dire plus techniques et ne considèrent pas que le mot se réfère à la question de l'existence et de la nature des dieux mais aux dieux eux-mêmes : l'adelotes ferait allusion au fait que les dieux ne s'offrent pas à l'expérience sensible. On peut citer par exemple Diels qui propose « Nichtwahrnehmbarkeit $»^{91}$, Mario Untersteiner « impossibilità di una loro esperienza sensibile $\gg^{92}$, Jean-Paul Dumont «leur invisibilité » ${ }^{93}$, Paul Demont et Monique Trédé « absence d'évidence ${ }^{94}$. Si on traduit le terme dans cette acception, Protagoras semblerait se référer au langage philosophique des présocratiques où la

87. Migliori-Ramelli-Reale 2006 p. 1576.

88. Solana Dueso 2013 p. 111.

89. Schirren \& Zinsmaier 2003 p. 37.

90. L'anecdote se trouve également en version grecque dans la soi-disant Theosophia de Tübingen. Hiéron est remplacé par un tıৎ (quelqu’un) plus indéfini (II, 24, 143-145, p. 35

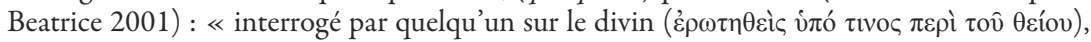
Simonide différa sa réponse pendant plusieurs jours et, interrogé par la suite sur la cause de ce report, il répondit : "plus je réfléchis sur le divin, plus je suis éloigné de quelque savoir que ce

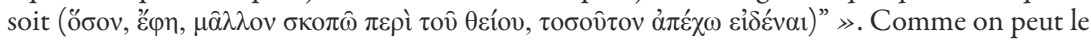
remarquer, dans cette version, le mot adelotes n'apparaît malheureusement pas.

91. Diels \& Kranz 1951-1952 p. 265.

92. Untersteiner 1961 p. 81.

93. Dumont 1969 p. 46.

94. Demont \& Trédé 1993 p. 158. 
notion d'adelon joue un rôle important, plus particulièrement chez Anaxagore - il suffit de penser à la célèbre sentence du fr. 59 B 21a DK = 25 D 6

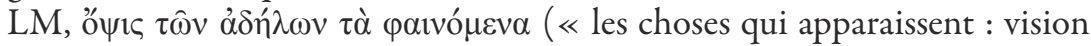
des choses invisibles $\gg$, trad. Laks $\&$ Most) et chez les atomistes ${ }^{95}$. Le terme adelon apparaît également en relation avec Protagoras dans un témoignage tardif mais significatif de Didyme l'Aveugle (In ps. 34, $17=\mathrm{R} 27 \mathrm{LM}$ ), conservé dans un papyrus retrouvé à Tura en Égypte (Pap. Texte Abh. III, 222, 21-22, p. 380 Gronewald 1969) ${ }^{96}$. De toute façon, à y regarder de plus près, les deux interprétations d'adelotes que les commentateurs proposent ne sont pas si éloignées : on pourrait dire que l'obscurité de la question est intrinsèquement liée au fait qu'une expérience sensible des dieux n'est pas possible selon Protagoras.

Comme nous avons déjà eu l'occasion de le rappeler, il n'est pas facile de reconstruire la manière dont le texte aurait pu se développer à la suite de l'incipit : certes, le $\mu \varepsilon^{\prime} v$ pourrait faire penser à une opposition, probablement à l'antithèse traditionnelle homme/dieu. Le texte aurait pu continuer en considérant les hommes, pour analyser le rapport des hommes avec les dieux, peut-être le problème du culte, peut-être le rôle de la religion dans le développement de la société humaine ${ }^{97}$.

Restons pour l'instant encore sur le texte du fr. 4. Comme on peut le

95. Pour une histoire de l'interprétation du fragment, $c f$. Wolbergs 2012. Pour ce qui est de la notion d'adelon chez Démocrite, surtout pour ce qui concerne son rapport avec la réflexion de Protagoras, d'une part, et la canonique d'Épicure, d'autre part, voir Lee 2005 p. 233-250.

96. Le texte attribue à Protagoras une position qui anticiperait l'epoche sceptique :

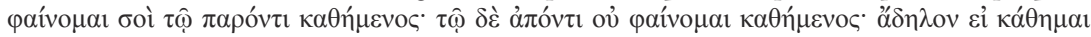
ì oủ Ká $\theta \eta \mu \alpha 1$ ( «à toi qui es présent, j’apparais assis ; à celui qui est absent, je n’apparais pas assis : il n'est pas clair que je suis assis ou que je ne suis pas assis »; trad. Laks-Most). Pour les principaux problèmes exégétiques, $c f$. Gagarin 2002.

97. Cf. Bonazzi 2010 p. 137-138. Comme le souligne Drozdek 2007 p. 109-113, il est naturel de penser au rôle que la religion joue dans le mythe de Prométhée du Protagoras (322d $=80 \mathrm{C} 1 \mathrm{DK}=31 \mathrm{D} 40 \mathrm{LM})$. Dans le Cratyle (400d-401a) de Platon, selon Socrate, étant

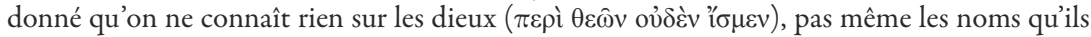
se donnent, la recherche doit s'orienter sur les hommes et sur l'opinion qu'ils pouvaient

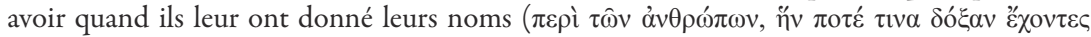

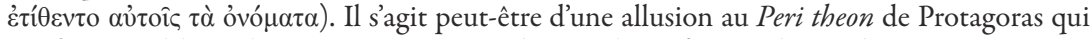
confirmerait l'hypothèse que nous venons de rappeler. Cf. Corradi 2012b p. 169-170. Dans ce contexte, il est également possible de trouver une allusion à la position de Protagoras dans un passage de Plutarque (Per. 8, 9), dans lequel le biographe, en reprenant Stésimbrote (FGH $107 \mathrm{~F} 9$ ), cite un passage du discours funèbre que Périclès prononça en l'honneur des soldats

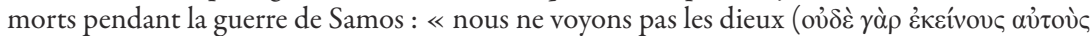

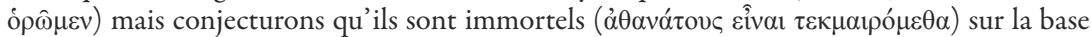
des honneurs que nous leur rendons et des biens que nous recevons d'eux. » Une conception analogue semble être détournée dans une perspective comique dans le prologue des Cavaliers d'Aristophane (30-35) : un des deux esclaves propose comme tekmerion de l'existence des dieux la haine qu' ils nourrissent contre lui. 
remarquer, Protagoras construit l'incipit de son Peri theon sur une série de motifs, traditionnels dans la littérature qui le précède, dont il était un grand connaisseur. Il n'y a pas lieu de rappeler ici l'ensemble des témoignages qui attestent l'intérêt que Protagoras portait à la poésie. Il suffit de rappeler ce que Platon lui fait dire dans le dialogue éponyme à propos du rôle fondamental de la compétence en poésie dans la paideia $(80 \mathrm{~A} 25 \mathrm{DK}=31 \mathrm{D} 31 \mathrm{LM})^{98}$. Comme on le sait, le thème de la difficulté de connaître le divin est un topos de la tradition littéraire et philosophique. Même si dans les épopées homériques, l'expérience de l'épiphanie du dieu est assez répandue, dans l'Odyssée (XVI, 161), on remarque que les dieux ne se rendent pas manifestes aux yeux de tous

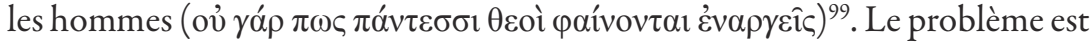
particulièrement prégnant dans les célèbres vers du fr. 21 B $34 \mathrm{DK}(=8 \mathrm{D} 49$ LM) de Xénophane dans lesquels le poète déclare qu'aucun homme n'a eu

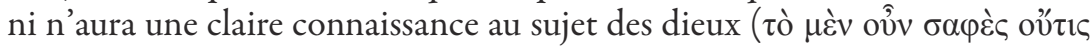

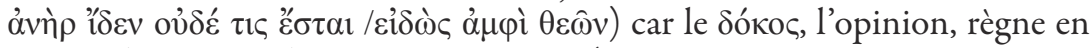
toutes choses ${ }^{100}$. Selon Diogène Laërce (IX, $24=30$ A 1 DK = 21 R 27 LM), Mélissos disait, à propos des dieux, qu'il ne faut pas se prononcer $(\pi \varepsilon \rho \grave{i}$

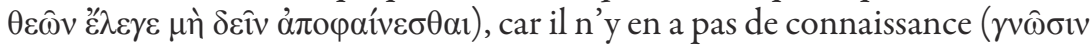
$\alpha \hat{\tau} \hat{\omega} v)^{101}$. Et Héraclite lui-même (22 B 5 DK $=9$ D 15 LM) critiquait ceux qui adressent leurs prières à des statues, comme quelqu' un qui s'entretiendrait

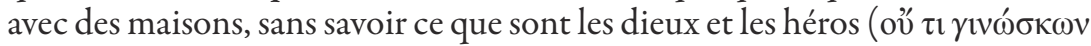
$\theta \varepsilon$ ov̀ ở $\delta$ ' ท̆ peut pas se faire au travers de la vue et du toucher $(31 \mathrm{~B} 133 \mathrm{DK}=22 \mathrm{D} 9$ LM ${ }^{103}$. Le thème de la difficulté qu'il y a à connaître le divin se mêle chez Protagoras à celui de la brièveté de la vie humaine. Naturellement, même dans ce cas, il n'est pas possible de faire abstraction d'Homère qui qualifie le personnage d'Achille de $\mu \iota v v v \theta \alpha ́ \delta ı c$ («à la courte existence », Il. 1, 352).

98. Je renvoie à ce propos à Corradi 2012b p. 133-175. Cf. maintenant aussi Andolfi 2014 p. 121-131.

99. Cf. Henrichs 2011 p. 19 et Versnel 2011 p. 37-43. Dans l'Odyssée (8, 201), les Phéaciens connaissent par exemple toujours ce privilège. Comme le souligne à juste titre Clay 1983 p. 171-172, il s'agit d'une caractéristique propre à des peuples aux marges du monde, en tant que reste d'un âge d'or dans lequel le contact entre les hommes et les dieux était plus simple.

100. Sur ce texte et sur les divers problèmes exégétiques le concernant, cf. Sassi 2011 p. 7-10 avec son ample bibliographie.

101. Pour ce qui concerne le rapport de l'affirmation de Mélissos avec la réflexion plus générale du philosophe sur l'être, $c f$. Mansfeld 2016 p. 81.

102. Sur l'ampleur de la critique de la religion traditionnelle qu'Héraclite développe dans ce texte, cf. l'analyse équilibrée de Fronterotta 2013 p. 355-356 n. 18.

103. Selon Picot 2012 p. 10 n. 34 la divinité en question serait le Sphairos. Cf. Primavesi 2013 p. 694. Hors du domaine de la poésie, il n'est pas aisé de saisir quel rapport entretient avec Protagoras un passage du livre 2, 3 d'Hérodote, selon lequel, touchant les dieux, tous

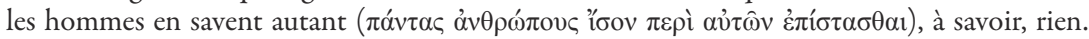
Cf. Lloyd 1989 p. 236. 
Le thème est repris par la lyrique archaïque : Mimnerme (fr. 2 West 1972 $=8$ Gentili-Prato) réinterprète la célèbre comparaison homérique entre les générations humaines et les feuilles d'Il. 6, 146-149 pour mettre en évidence la nature éphémère de l'homme. Dans un contexte qui fait explicitement allusion au même passage de l'Iliade, le fr. 8 West 1972 (deest Gentili-Prato), que Stobée $(4,34,28)$ attribue à Simonide, stigmatise la sottise des jeunes gens qui ne comprennent pas combien le temps de la jeunesse et de la vie

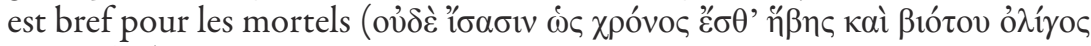
$\theta v \eta \tau$ oîs $)^{104}$. Le thème de la brièveté de la vie se trouve déjà mis en relation avec les limites épistémologiques de l'homme chez Empédocle (31 B 2 DK

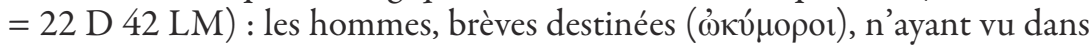

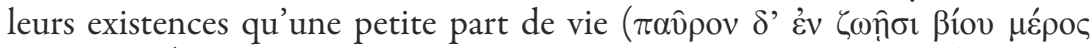
$\alpha \theta \rho \eta ́ \sigma \alpha \nu \tau \varepsilon \varsigma)$, ne parviennent qu'à un savoir aléatoire, convaincus de la seule chose que chacun se trouve avoir rencontrée ${ }^{105}$. Pour ce qui est du rapport entre le temps et la connaissance, il est possible de citer Xénophane (21 B 18 $\mathrm{DK}=8 \mathrm{D} 53 \mathrm{LM}$, trad. Laks \& Most) : « ce n'est pas que dès le début ( $\alpha \dot{\pi}$ '

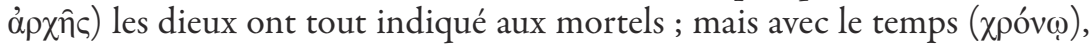
ils trouvent plus et mieux en cherchant $\gg{ }^{106}$.

En ce qui concerne la question de la forme du dieu, Protagoras joue également avec des motifs traditionnels. L'ample typologie des épiphanies divines dans les poèmes homériques montre que les dieux avaient la possibilité de se manifester sous des formes diversifiées, à la fois sous forme de voix, d'un être humain déterminé ou d'un animal. Parfois, ils peuvent apparaître entourés de brume. Parfois ils peuvent enfin révéler leur taille et leur beauté réelles. Même pour ce qui est de ce thème, le point de vue de Xénophane est fondamental. Il suffit de penser à la série de fragments critiquant l'anthropomorphisme des

104. Sur le thème d'Achille $\mu$ tvvv $\theta \alpha ́ \delta 1 o s$ dans l'Iliade, cf. Garcia 2013 p. 11-14. Pour ce qui est de la célèbre analogie entre les races humaines et les feuilles, on trouvera une ample analyse

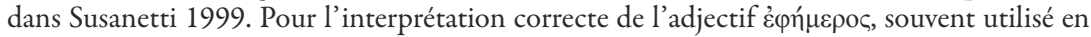
poésie pour décrire la nature de l'homme, la recherche de Fränkel 1946 est encore très importante. Sans aucun doute, dans la tradition que Protagoras réélabore pour l'incipit du Peri theon, l'opposition entre le caractère caduc de la vie humaine et l'éternité de la vie des dieux joue un rôle essentiel. Cf. Dreßler 2010 p. 33.

105. On trouve une analyse approfondie de ce fragment dans Gheerbrant 2017 p. 39-98. Pour ce qui est des rapports entre la durée de la vie humaine et les possibilités cognitives de l'homme, il faut prendre également en considération le célèbre apophtegme hippocratique

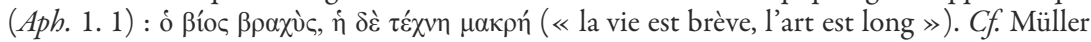
1976 p. 320 n. 32 . La réflexion sur le rapport entre brièveté de la vie et critique rationaliste de la religion traditionnelle, qu'Euripide développe dans le premier stasimon des Bacchantes (395-397), cacherait, selon Di Benedetto 2004 p. 8-9, une polémique contre Protagoras.

106. Sur les nombreux problèmes exégétiques concernant le fragment, $c f$. Lesher 1992 p. $149-155$. 
dieux homériques $(21 \mathrm{~B} 15 \text { et } 16=8 \mathrm{D} 14 \text { et } 13 \mathrm{LM})^{107}$. La question revient dans un célèbre passage d'Hérodote $(2,53)$ dans lequel, comme l'a remarqué Walter Burkert, il est possible de repérer une influence de la position protagoréenne : nous avons déjà cité à ce propos le parallèle entre la séquence

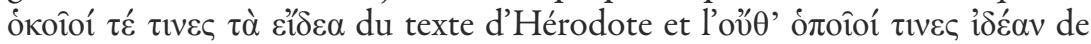
Protagoras. Selon l'historien, les Grecs devraient leur panthéon aux Pélasges qui, à leur tour, l'auraient tenu des Égyptiens. Auparavant, les Pélasges sacrifiaient à des divinités anonymes. De quels parents chacun des dieux naquit, ou si tous existèrent de tout temps, quels ils sont quant à leur forme (ókoîó

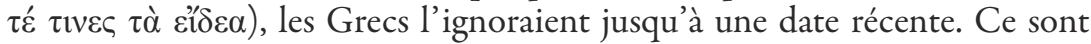
Homère et Hésiode qui, dans leurs poèmes, ont fixé pour les Grecs une théogonie, attribué aux dieux leurs qualificatifs, partagé entre eux les honneurs et les compétences et tracé leurs figures (

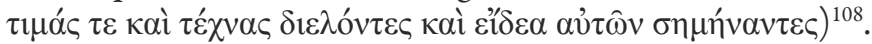

Comme cela ressort de ce bref répertoire de passages parallèles, Protagoras s'insère dans une tradition bien consolidée de réflexion littéraire et philosophique tout en proposant un point de vue nouveau : les difficultés épistémologiques relatives à la connaissance du divin, qui émergent dans la réflexion présocratique, et la conscience des limites de l'homme, propre à tout un courant pessimiste de la tradition poétique, amènent le sophiste à proclamer son propre scepticisme à l'égard de la possibilité de savoir si les dieux existent ou non et de déterminer leur forme, à cause d'une série d'obstacles épistémologiques. Parmi ces obstacles, Protagoras en sélectionne deux, l'un qui concerne l'objet, le divin, par nature obscur pour les hommes, l'autre qui concerne le sujet, l'homme, naturellement caractérisé par un temps de vie limité qui ne lui permet pas de parvenir à un résultat certain sur la question.

Après avoir essayé d'insérer le Peri theon dans le cadre de la tradition philosophique et littéraire du passé, il est possible maintenant de l'inscrire dans le cadre de la réflexion protagoréenne elle-même. Pour ce faire, il est évidemment tout à fait naturel de le mettre en relation avec le principe de l'homme-mesure (80 B $1 \mathrm{DK}$ ). Comme on le sait, selon ce principe, Protagoras considérait l'homme comme le critère de la vérité. L'homme est la mesure de toutes choses : à propos des choses qui sont, c'est-à-dire qui se manifestent à lui, il dit, il juge, il pense qu'elles sont, existent, sont vraies.

107. Cf. Sassi 2013 p. 284-294. Drozdek 2005 p. 42 insiste à juste titre sur le rapport entre le problème des métamorphoses des dieux dans le mythe et l'aporie de Protagoras à propos de leur forme. Pour la question de la morphologie des dieux, dans le cadre d'une recherche plus ample concernant les épiphanies dans la culture et dans la littérature grecques, $c f$. Petridou 2015 p. $29-105$.

108. Cf. Burkert 1985 p. 131. Néanmoins, Di Benedetto 2007a p. 1522-1523 n’exclut pas que ce soit Protagoras qui s'inspirerait d'Hérodote. Provencal 2015 p. 43-45 met clairement en évidence les analogies et les différences qui subsistent entre les positions des deux intellectuels ioniens à propos du problème de la connaissance des dieux. 
À propos de celles qui ne sont pas, c'est-à-dire qui ne se manifestent pas à lui, il dit, il juge, il pense qu'elles ne sont pas, n'existent pas, ne sont pas vraies. Il tient pour vrai ce qui lui apparaît et les opinions qu'il se forme sur cette base. Ce qui lui apparaît comme vrai, réel, doté de certaines qualités déterminées est pour lui vrai, réel, doté desdites qualités. Ce qui ne lui apparaît pas n'est pour lui ni vrai ni réel. Les qualités qu'il ne perçoit pas dans un certain objet n'appartiennent pas pour lui audit objet ${ }^{109}$. Dans le cas des dieux, Protagoras, qui s'exprime, conformément à la perspective de l'homme-mesure, à la première personne du singulier, n'a pas une expérience directe qui lui permettrait d'établir si les dieux existent ou non. Néanmoins, il faut remarquer que cette absence d'expérience directe ne conduit pas Protagoras à la négation de l'existence des dieux mais, en anticipant l'epoche des sceptiques ${ }^{110}$, à se contenter de constater l'impossibilité de parvenir à la solution du problème. La présence d'un décalage entre la perspective de l'homme-mesure et celle du Peri theon a été mise en évidence de manière convaincante par Annie Hourcade ${ }^{111}$. Certes, il est possible d'évoquer la nature particulière de l'objet en question, les dieux, et d'interpréter, par conséquent, la connaissance des dieux comme la connaissance d'un savoir objectif qui échappe aux possibilités de l'homme, un type de savoir qui est par nature exclu du domaine d'application de l'homme-mesure ${ }^{112}$. Mais, en fin de compte, il est peut-être possible de réunifier la perspective des deux écrits selon un autre point de vue. Dans un travail de 2007, dont les résultats ont été repris dans mon volume de 2012, j'avais cherché à donner une interprétation du principe de l'homme-mesure par rapport à la tradition poétique de l'incipit ${ }^{113}$. J'interprétais l'homme-mesure comme le renversement d'une tradition qui plaçait dans l'incipit des textes poétiques l'invocation à la Muse comme garantie de la vérité du chant. La mesure, le metron, du savoir n'est plus, dans la perspective du sophiste, un don de la divinité au poète mais elle devient, avec Protagoras, une possession propre à l'homme. Certes, il s'agit d'un savoir d'une tout autre nature, un savoir relatif, humain ; ce n'est donc

109. Pour ce qui est de l'interprétation du célèbre principe de Protagoras, je me permets de renvoyer à Corradi 2012b p. 118-124 avec une discussion de la bibliographie. Cf. maintenant Gavray 2017 p. 23-29.

110. Sur les rapports entre Protagoras et le scepticisme, $c f$. Decleva Caizzi 1976 p. $439-441$ et Lee 2010 p. 19-22.

111. Hourcade 2009 p. 43-47. Dans les Bacchantes (199-203 = 80 C 4 DK), Euripide semble présupposer un rapport étroit entre l'Aletheia, connue également sous le titre de Kataballontes, et la réflexion du Peri theon. Dans la pièce, Tirésias et Cadmos soutiennent qu'ils ne se comportent pas comme des sophistes à l'égard des traditions religieuses les plus anciennes : aucun raisonnement ne les renversera, $\kappa \alpha \tau \alpha \beta \alpha \lambda \varepsilon \hat{\text {, }}$ même pas le savoir trouvé par les plus profonds esprits. Cf. Di Benedetto 2004 p. 354-356.

112. Cf. par exemple Zilioli 2007 p. 84-85.

113. Corradi 2007 et Corradi 2012b p. 112-130. 
pas par hasard que Platon, dans les Lois (IV 716c = 31 R 10 LM), joue sur l'opposition entre homme-mesure et dieu-mesure ${ }^{114}$.

Le Peri theon développe dans son incipit une réflexion qui s'harmonise parfaitement avec cette perspective : les limites de la vie de l'homme ne permettent pas une expérience du divin et ne permettent donc pas d'établir s'il existe. Il n'y a pas de place pour la rencontre du poète avec la Muse de la tradition $^{115}$, les dieux sont en effet exclus de l'horizon cognitif de l'homme. Comme l'incipit de l'Aletheia, l'incipit du Peri theon peut donc être lu comme la réponse de Protagoras à une tradition poétique qui considérait l'incipit comme le lieu privilégié de la réflexion sur le rapport entre l'homme et un savoir objectif, don de la Muse. Cette réponse permettait à Protagoras d'affirmer le caractère humain du savoir. Ce n'est peut-être pas par hasard non plus que, dans le texte « protagoréen »d'Hérodote que nous venons d'évoquer $(2,52)$, il est fait allusion, d'une façon qui n'est peut-être pas dépourvue d'ironie, à Homère et à Hésiode qui auraient tout récemment révélé aux Grecs la forme des dieux ${ }^{116}$. Il ne faut pas exclure que, dans le sillage de Xénophane, Protagoras, dans le Peri theon, ait mis en évidence les inconséquences de la conception théologique des poètes en développant sa propre argumentation dans un dialogue avec la tradition littéraire.

Malgré les doutes de von Fritz ${ }^{117}$, il me semble donc que le sophiste pouvait sans dommage développer aisément son traité à la suite de son scandaleux incipit. D'ailleurs, le thème des dieux n'était pas isolé dans le cadre de la réflexion de Protagoras mais s'insérait dans une perspective plus ample qui concernait également le problème de l'au-delà. Dans le catalogue des écrits du sophiste, se trouve en effet un Peri ton en Haidou (80 B 8h DK = 31 D 1 LM). Malheureusement nous n'avons aucun renseignement sur son contenu. Néanmoins, la reprise de la Nekuia homérique que Platon propose dans la description qu'il donne des sophistes dans la maison de Callias dans le

114. Sur la signification de ce si complexe passage platonicien, $c f$. au moins van Riel 2013 p. 17-19.

115. Sur les scènes de l'investiture poétique de la part des Muses, l'étude de Kambylis 1965 est désormais canonique. Pour la tradition rationalisante qui, après Hésiode, tend à placer ce type de scène dans une dimension onirique, cf. Tulli 2008.

116. Il faut remarquer que le passage d'Hérodote renvoie, comme le Peri theon de Protagoras, à la question de la temporalité en relation avec le problème de la recherche théologique. Une relation entre la question de l'existence des dieux et la durée de la vie humaine apparaît également dans le cadre de la critique des penseurs athées du livre X des Lois (888a-d). Selon l'Athénien, le temps a la capacité de faire changer d'opinion à propos de cette question : aucun

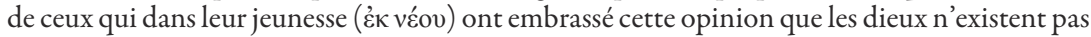

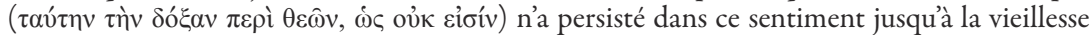
( $\left.\pi \rho \mathrm{c}_{\varsigma} \gamma \hat{\eta} \rho \alpha \varsigma\right)$. Ne s'agirait-il pas d'une allusion polémique à la position de Protagoras ? Pour le rapport du passage des Lois avec Platon $R$. 330d-e, cf. Schöpsdau 2011 p. 383-384.

117. Fritz 1957 col. 920. 
Protagoras (314e-316a) pourrait avoir un rapport avec l'écrit protagoréen ${ }^{118}$. Sur la base de cette hypothèse, on pourrait penser que Protagoras dans ce texte aurait pu analyser la célèbre page homérique. Il est également possible de supposer qu'à propos de l'existence de l'au-delà, le sophiste soit parvenu à la même conclusion sceptique qu'il trouvait à propos du problème de l'existence et de la forme des dieux. Il n'est même pas à exclure que, même dans le cas des choses de l'Hadès, Protagoras ait fait allusion à son adelotes ${ }^{119}$. Naturellement, même dans ce cas, nous devons nous contenter de formuler des hypothèses. Quoi qu'il en soit, nos analyses ont permis de parvenir à une meilleure compréhension d'un point de vue à la fois critico-textuel et exégétique des restes du Peri theon sauvés du naufrage des textes de Protagoras. Grâce à elles, il a été en effet possible de formuler des hypothèses plausibles sur les origines de la tradition concernant la persécution judiciaire que le sophiste aurait subie suite à la lecture publique de son ouvrage et sur son historicité ainsi que, de manière plus générale, de restituer au Peri theon son rôle capital dans la pensée du sophiste et dans la réflexion littéraire et philosophique des Grecs sur les dieux.

118. Sur la célèbre description de la maison de Callias, je renvoie à Corradi 2014 p. 35-39.

119. Le terme adelotes, comme nous l'avons rappelé, renvoie à l'au-delà chez Plutarque fr. 178 Sandbach. Comme l'a souligné, à la suite de Sassi 1987, Casertano 2015 p. 335, 384 et 390, une série de passages du Phédon (85a-b, 107a-b et surtout 108d-e) semble rappeler plus ou moins explicitement l'incipit du Peri theon de Protagoras. À y regarder de près, dans tous ces passages, la prudence épistémologique que Protagoras appliquait au problème de l'existence et de la forme des dieux est utilisée par Platon en relation avec le thème de l'au-delà. On pourrait formuler l'hypothèse selon laquelle, dans le Phédon, Platon se réferrerait en fait audit Peri ton en Haidou, dans lequel le sophiste aurait pu exposer ses doutes quant à l'existence et à la nature de l'au-delà d'une manière tout à fait analogue à ce qu'il disait à propos des dieux dans le Peri theon. Sur la prudence épistémologique de Platon dans le domaine théologique, cf. van Riel 2013 p. 30-34. On pourrait peut-être discerner un rapport avec les deux écrits de Protagoras dans la République, plus particulièrement dans l'analyse des représentations poétiques des dieux et de l'Hadès (379a-387c) : selon une tradition remontant à Aristoxène (67 Wehrli $=80$ B 5 DK $=31 \mathrm{R} 1 \mathrm{LM}$ ), dans la République, Platon aurait repris les Antilogies de Protagoras, cf. Corradi 2016 p. 336-337. 


\section{BIBLIOGRAPHIE}

ANDolfi, I. 2014 : «Una vetrina esegetica per tre sofisti. Il carme di Simonide nel Protagora di Platone », Seminari Romani di Cultura Greca, 3/1 (2014), p. 117-149.

Arrighetti, G. 2008 : « Cameleonte peripatetico e gli studi sulla biografia greca », dans P. Arduini, S. Audano, A. Borghini, A. Cavarzere, G. Mazzoli, G. Paduano \& A. Russo (éd.), Studi offerti ad Alessandro Perutelli, vol. 1, Roma, 2008, p. 63-69.

Barnes, J. 2015 : « Protagoras the Atheist ? », dans Essays in Ancient Philosophy, IV : Mantissa, textes réunis par M. Bonelli, Oxford, 2015, p. 190-203.

Beatrice, P. F. 2001 (éd.) : Anonymi monophysitae Theosophia : an Attempt at Reconstruction, Leiden, 2001 (Supplements to Vigiliae Christianae, 56).

Beresford, A. 2013 : « Fangs, Feathers, \& Fairness : Protagoras on the Origins of Right and Wrong », dans J. M. van Ophuijsen, M. van Raalte \& P. Stork (éd.), Protagoras of Abdera: The Man, His Measure, Leiden-Boston, 2013, p. 139-162.

Betegh, G. 2009: « Tale, Theology and Teleology in the Phaedo », dans C. Partenie (éd.), Plato's Myths, Cambridge-New York, 2009, p. 77-100.

BodéÜs, R. 1987 : « Réflexions sur un court propos de Protagoras », Les Études classiques, 55 (1987), p. 241-257.

Bolonyai, G. 2007 : « Protagoras the Atheist », Rhizai, 4/2 (2007), p. 247-269.

BonazzI, M. 2007 : «Protagora e Seniade », dans F. Trabattoni \& M. Bonazzi, I sofisti, Milano, 2007, p. 89-141 (BUR. Classici greci e latini).

— 2009 (trad.) : « Protagoras d'Abdère », dans J.-F. Pradeau (éd.), Les Sophistes, 1, Paris, 2009, p. $45-90$ et p. 443-472.

- 2010 : I sofisti, Roma, 2010 (Pensatori, 18).

- 2012 : « Il mito di Prometeo nel Protagora : una variazione sul tema delle origini », dans F. Calabi \& S. Gastaldi (éd.), Immagini delle origini : la nascita della civiltà e della cultura nel pensiero greco e romano, Sankt Augustin, 2012, p. 41-57 (Collegium politicum, 5).

Boudon-Millot, V. \& A. Pietrobelli 2005 (éd.) : « Galien ressuscité : édition princeps du texte grec du De propriis placitis », Revue des études grecques, 118/1 (2005), p. 168-213.

BrancaCci, A. 2002 : « Protagora e la Techne sophistike. Plat. Prot.316D-317C », Elenchos, 23/1 (2002), p. 11-32.

Bremmer, J. N. 2007 : « Atheism in Antiquity », dans M. Martin (éd.), The Cambridge Companion to Atheism, Cambridge, 2007, p. 11-16 (Cambridge Companions to Philosophy).

BRISSON, L. 1975: «Le mythe de Protagoras : essai d'analyse structurale », Quaderni urbinati di cultura classica, 20 (1975), p. 7-37.

Burkert, W. 1985 : « Herodot über die Namen der Götter : Polytheismus als historisches Problem », Museum Helveticum, 42 (1985), p. 121-132.

CAPrA, A. $2000:$ : Platone e la storia : la fine di Protagora e lo statuto letterario dei dialoghi socratici », Acme, 53/2 (2000), p. 19-37.

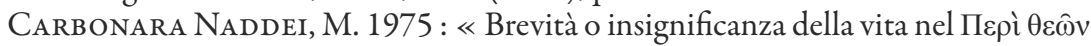


di Protagora ? », Atti dell'Accademia di Scienze Morali e Politiche della Società Nazionale di Scienze, Lettere ed Arti di Napoli, 86 (1975), p. 465-503.

Casertano, G. 2015 (trad.) : Platone, Fedone, o dell'anima : dramma etico in tre atti, traduzione, commento e note, Napoli, 2015 (Philosophike skepsis, 2).

Strauss, J. C. 1983 : The Wrath of Athena : Gods and Men in the Odyssey, Princeton, 1983.

Conte, G. B. 2012 : Memoria dei poeti e sistema letterario : Catullo, Virgilio, Ovidio, Lucano, Palermo, 2012 (La Nuova diagonale, 96).

Corradi, M. 2007 : « L'origine della tradizione sul processo di Protagora », dans M. Erler \& S. Schorn (éd.), Die griechische Biographie in hellenistischer Zeit : Akten des internationalen Kongresses vom 26.-29. Juli 2006 in Würzburg, Berlin-New York, 2007, p. 285-301 (Beiträge zur Altertumskunde, 245).

- 2012a : «Thucydides adoxos and Praxiphanes », dans A. Martano, E. Matelli \& D. Mirhady (éd.), Praxiphanes of Mytilene and Chamaeleon of Heraclea, text, translation, and discussion, New Brunswick, 2012, p. 495-523 (Rutgers University Studies in Classical Humanities, 18.)

- 2012b : Protagora tra filologia e filosofia : le testimonianze di Aristotele, Pisa-Roma, 2012 (Biblioteca di studi antichi, 96).

- 2014: «Platone al termine del Protagora: la profezia di una paideia possibile », dans M. Vallozza (éd.), Etica e politica : tre lezioni su Platone: Atti del Convivium Viterbiense 2013 (Viterbo, 17 maggio 2013), Viterbo, 2014, p. 33-52.

- 2015: « Gorgia di Leontini, Su ciò che non è, Hildesheim-Zürich-New York 2010 (testo greco, trad. e comm. a c. di R. Ioli) », Eikasmos, 26 (2015), p. 445-457.

- 2016: « Doing Business with Protagoras (Prot.313e) : Plato and the Construction of a Character », dans G. Cornelli (éd.), Plato's Styles and Characters : Between Literature and Philosophy, Berlin, 2016, p. 335-356 (Beiträge zur Altertumskunde, 341).

— 2017 : « Il Пвpì $\theta \varepsilon \hat{\omega} v$ di Protagora : un nuovo tentativo di ricostruzione », Maia. Rivista quadrimestrale di letterature classiche, n.s. 69/3 (sett.-dic. 2017), p. 444-469.

De Falco, V. 1923 (éd.) : L'Epicureo Demetrio Lacone, Napoli, 1923 (Biblioteca filologica classica, 11).

Decleva Caizzi, F. 1976: « La tradizione protagorea ed un frammento di Diogene di Enoanda », Rivista di filologia e di istruzione classica, 104 (1976), p. 435-442.

Demont, P. \& M. Trédé 1993 (trad.) : Platon, Protagoras, traduction nouvelle, introduction et commentaires, Paris, 1993 (Le Livre de Poche, 4616).

Derenne, E. 1930 : Les procès d'impiété intentés aux philosophes à Athènes au $V^{e}$ et au $I V^{e}$ siècles avant J.-C., Liège-Paris, 1930 (Bibliothèque de la Faculté de Philosophie et de Lettres de l'Université de Liège, 45).

Di Benedetto, V. 2004 (éd.) : Euripide, Le Baccanti, appendice metrica di Ester Cerbo, nuov. ed. ampliata, Milano, 2004 (BUR. Classici greci et latini).

- 2007a : « Contributo al testo del frammento di Protagora sugli dei », dans $I l$ richiamo del testo : contributi di fllologia e letteratura, prefazione di Riccardo Di Donato, vol. 4, Pisa, 2007, p. 1521-1523.

- 2007b : « Protagora ed Euripide », dans Il richiamo del testo : contributi difilologia eletteratura, prefazione di Riccardo Di Donato, vol. 4, Pisa, 2007, p. 1515-1520.

Diels, H. \& W. Kranz 1951-1952 (éd.) : Die Fragmente der Vorsokratiker, 1-3, 
Berlin, 1951-1952.

Dietz, K.-M. 1976 : Protagoras von Abdera : Untersuchungen zu seinem Denken, Bonn, 1976 (Habelts Dissertationsdrucke. Reihe klassische Philologie, 25).

Di Marco, M. 1989 (éd.) : Timone di Fliunte, Silli, introduzione, edizione critica, traduzione e commento, Roma, 1989 (Testi e commenti, 10).

Dodds, E. R. 1951 : The Greeks and the Irrational, Berkeley-Los Angeles, 1951 (Sather Classical Lectures, 25).

Dorandi, T. 2013 (éd.) : Diogenes Laertius, Lives of Eminent Philosophers, Cambridge, 2013 (Cambridge Classical Texts and Commentaries, 50).

Dover, K. 1976 : « The Freedom of the Intellectual in Greek Society », dans The Greeks and Their Legacy: Collected Papers, 2, Prose Literature, History, Society, Transmission, Influence, Oxford-New York, 1988, p. 135-158.

Dressler, J. 2010 : Philosophie vs. Religion ? Die Asebie-Verfahren gegen Anaxagoras, Protagoras und Sokrates im Athen des füften Jahrhunderts v. Chr., Norderstedt, 2010.

- 2014: Wortverdreher, Sonderlinge, Gottlose: Kritik an Philosophie und Rhetorik im klassischen Athen, Berlin-Boston (Mass.), 2014 (Beiträge zur Altertumskunde, 331).

Drozdek, A. 2005 : « Protagoras and Instrumentality of Religion », L' Antiquité classique, 74 (2005), p. 41-50.

- 2007 : Greek Philosophers as Theologians: The Divine Arche, Aldershot, 2007.

Dumont, J.-P. 1969 (trad.) : Les sophistes, Fragments et témoignages, Paris, 1969 (SUP. Les Grands Textes).

Dyck, A. R. 2003 (éd.) : Cicero, De Natura Deorum : Liber I, Cambridge-New York, 2003 (Cambridge Greek and Latin Classics).

ERLER, M. 2007 : « Biographische Elemente bei Platon und in hellenistischer Philosophie », dans M. Erler \& S. Schorn (éd.), Die griechische Biographie in hellenistischer Zeit : Akten des internationalen Kongresses vom 26.-29. Juli 2006 in Würzburg, Berlin-New York, 2007, p. 11-24 (Beiträge zur Altertumskunde, 245).

FAHR, W. 1969 : EEOY $\Sigma$ NOMIZEIN : Zum Problem der Anfänge des Atheismus bei den Griechen, Hildesheim, 1969 (Spudasmata, 26).

FIlonik, J. 2013 : « Athenian Impiety Trials : A Reappraisal », Dike, 16 (2013), p. 11-96.

Fortenbaugh, W. W. \& E. E. Schütrumpf 2000 (éd.) : Demetrius of Phalerum, Text, translation, and discussion, New Brunswick (N.J.), 2000 (Rutgers University Studies in Classical Humanities, 9).

FränKel, H. 1946 : « Man's "Ephemeros" Nature According to Pindar and Others », Transactions and Proceedings of the American Philological Association, 77 (1946), p. 131-145.

FrITZ, K. von 1957 : « Protagoras 1 », Paulys Realencyclopädie der classischen Altertumswissenschaft, XXIII, 1, col. 908-921.

Fronterotta, F. 2013 : Eraclito, Frammenti, Milano, 2013 (BUR. Classici greci e latini).

Gagarin, M. 2002 : «Protagoras' New Fragment : Thirty Years Later », dans B. Amden, P. Flensted-Jensen, T. Heine Nielsen, A. Schwartz \& C. Gorm Tortzen (éd.), Noctes Atticae : 34 articles on Graeco-Roman Antiquity and Its 
Nachleben: Studies Presented to Jorgen Mejer on His Sixtieth Birthday March 18, 2002, Copenhagen, 2002, p. 114-120.

Garcia, L. F. 2013 : Homeric Durability : Telling Time in the Iliad, Cambridge (Mass.), 2013 (Hellenic Studies, 57 [58]).

Gavray, M.-A. 2017 : Platon, héritier de Protagoras : dialogue sur les fondements de la démocratie, Paris, 2017 (Tradition de la pensée classique).

GazZano, F. 2014 : «Sovrani "barbari” e santuari greci : qualche riflessione », dans L. R. Cresci (éd.), Spazio sacro e potere politico in Grecia e nel Vicino Oriente,

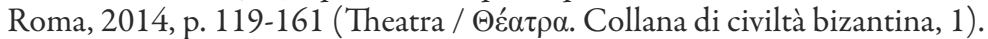

Gemelli Marciano, M. L. 2007 : « Lire du début : quelques observations sur les incipit des présocratiques », Philosopbie Antique, 7 (2007), p. 7-37.

Gheerbrant, X. 2017 : Empédocle, une poétique philosophique, Paris, 2017 (Kaïnon. Anthropologie de la pensée ancienne, 6).

Gigon, O. 1985 : « Il libro "Sugli dèi” di Protagora », Rivista critica di storia della filosofia, 40 (1985), p. 419-448.

Graham, D. W. 2010 : The Texts of Early Greek Philosophy : the Complete Fragments and Selected Testimonies of the Major Presocratics, Cambridge, 2010.

Grau, S. 2010 : « How to Kill a Philosopher : the Narrating of Ancient Greek Philosophers' Deaths in Relation to Their Way of Living », Ancient Philosophy, 30/2 (2010), p. 347-381.

Gronewald, M. 1969 (éd.) : Didymos der Blinde, Psalmenkommentar (Tura-Papyrus), teil III, Kommentar zu Psalm 29-34, Bonn, 1969 (Papyrologie Texte und Abhandlungen, 8).

Guthrie, W. K. C. 1969 : A History of Greek Philosophy, 3, The Fifth-Century Enlightment, Cambridge, 1969.

Henrichs, A. 1974 : « Die Kritik der stoischen Theologie im PHerc. 1428 », Cronache Ercolanesi, 4 (1974), p. 5-32.

- 1976: «The atheism of Prodicus », Cronache Ercolanesi, 6 (1976), p. 15-21.

- 2010 : «What Is a Greek God ? », dans J. N. Bremmer \& A. Erskine (éd.), The Gods of Ancient Greece : Identities and Transformations, Edinburgh, 2010, p. 19-39 (Edinburgh Leventis Studies, 5).

Hourcade, A. 2009 : Atomisme et sophistique : la tradition abdéritaine, Bruxelles, 2009 (Cahiers de philosophie ancienne, 21).

KaHn, Ch. H. 2003 : The Verb 'Be' in Ancient Greek : with a new introductory essay, Indianapolis, 2003.

Kaibel, G. 1887-1890 (éd.) : Athénée, Athenaei Naucratitae Dipnosophistarum libri XV, I-III, Leipzig, 1887-1890 (Bibliotheca scriptorum graecorum et romanorum teubneriana. Auctores graeci).

Kambylis, A. 1965 : Die Dichterweihe und ibre Symbolik : Untersuchungen zu Hesiodos, Kallimachos, Properz und Ennius, Heidelberg, 1965 (Bibliothek der Klassischen Altertumswissenschaften, 2. Reihe).

Kerferd, G. B. 1981 : The Sophistic Movement, Cambridge, 1981.

LAKs, A. \& G. W. Most 2016a (éd.) : Early Greek Philosophy, edited and translated, in collaboration with G. Journée and assisted by L. Iribarren \& D. Lévystone, 1-9, Cambridge, 2016 (Loeb Classical Library, 524).

- 2016b (éd.) : Les Débuts de la philosophie : des premiers penseurs grecs à Socrate, édition et traduction, avec la collaboration de G. Journée et le concours de 
L. Iribarren et D. Lévystone, Paris, 2016 (Ouvertures bilingues).

LeE, M.-K. 2005 : Epistemology after Protagoras : Responses to Relativism in Plato, Aristotle, and Democritus, Oxford, 2005.

- 2010 : « Antecedents in Early Greek Philosophy », dans R. Bett (éd.), The Cambridge Companion to Ancient Scepticism, Cambridge, 2010, p. 13-35 (Cambridge Companions to Philosophy).

Lefkowitz, M. R. 1987 : « Was Euripides an Atheist ? », Studi Italiani di Filologia Classica, $3^{\text {e s., }} 5$ (1987), p. 149-166.

- 2012: The Lives of the Greek Poets, Baltimore, 2012.

LENFANT, D. 2002 : «Protagoras et son procès d'impiété : peut-on soutenir une thèse et son contraire ? », Ktèma, 27 (2002), p. 135-154.

- 2009 (éd.) : Les Histoires perses de Dinon et d'Héraclide : fragments édités, traduits et commentés, Paris, 2009 (Persika, 13).

Lesher, J. 1992 (éd.) : Xenophanes of Colophon, Fragments, a text and translation with a commentary, Toronto, 1992 (Phoenix Supplementary vol. 30. The Phoenix Presocratics. 4).

Lloyd, A. B. \& A. Fraschetti 1989 (éd. et trad.) : Erodoto, Le storie, II, Libro 2 : L'Egitto, Milano, 1989 (Fond. Lorenzo Valla Scrittori greci \& latini).

Mansfeld, J. 1981 : « Protagoras on Epistemological Obstacles and Persons », dans G. B. Kerferd (éd.), The Sophists and Their Legacy : Proceedings of the Fourth International Colloquium on Ancient Philosophy held in cooperation with Projektgruppe Altertumswissenschaften der Thyssenstiftung at Bad Homburg, 29th August - 1st September 1979, Stuttgart, 1981, p. 38-53.

- 2016 : « Melissus between Miletus and Elea », dans M. Pulpito (éd.), Eleatica 2012 : Melissus between Miletus and Elea, Sankt Augustin, 2016, p. 71-114 (Eleatica, 5).

Manuwald, B. 1999 (trad.) : Platon, Werke : Übersetzung und Kommentar, 6, 2, Protagoras, Göttingen, 1999.

Matelli E. 2012 (éd.) : Prassifane, Testimonianze e frammenti : fllosofia e grammatica in età ellenistica, Milano, 2012 (Pubblicazioni del Centro di Ricerche di Metafisica. Temi metafisici e problemi del pensiero antico : studi e testi, 129).

Mayhew, R. 2011 (éd.) : Prodicus the Sophist, Texts, Translation, and Commentary, Oxford, 2011.

Meier, M. H. E. 1883 : « Diagoras », dans J.-S. Ersch \& J. G. Gruber (éd.), Allgemeine Encyclopädie der Wissenschaften und Künste, 1, Leipzig, 1883, p. 439-448.

Migliori, M., I. Ramelli \& G. Reale 2006 (trad.) : « C. Antica sofistica », dans G. Reale et al., I Presocratici, prima traduzione integrale con testi originali a fronte delle testimonianze e dei frammenti nella raccolta di $H$. Diels e W. Kranz, Milano, 2006, p. 1543-1863 (Bompiani Il pensiero occidentale).

Montanari, F. 2012 : «The Peripatos on Literature : Interpretation, Use and Abuse », dans A. Martano, E. Matelli \& D. Mirhady (éd.), Praxiphanes of Mytilene and Chamaeleon of Heraclea, New Brunswick, 2012, p. 339-358 (Rutgers University Studies in Classical Humanities, 18).

Most, G. 2012 : « Plato’s Exoteric Myths », dans C. Collobert, P. Destrée \& F. J. Gonzalez (éd.), Plato and Myth. Studies on the Use and Status of Platonic Myths, Leiden-Boston, p. 13-24 (Mnemosyne. Supplements, 337).

Müller, C. W. 1976 : «Protagoras über die Götter », dans C. J. Classen (éd.), 
Sophistik, Darmstadt, 1976, p. 312-340 (Wege der Forschung, 187).

Napolitano, M. 2012 (trad.) : I Kolakes di Eupoli, introduzione, traduzione, commento, Frankfurt am Main, 2012 (Studia comica, 4).

Obbink, D. 1996 (éd.) : Philodemus, On Piety, Part 1, Critical Text with Commentary, Oxford, 1996.

Petridou, G. 2015 : Divine Epiphany in Greek Literature and Culture, Oxford, 2015.

PiCCIRILli, L. 1997 : «Il primo caso di autodaféletterario : il rogo dei libri di Protagora $\gg$, Studi Italiani di Filologia Classica, $3^{\mathrm{e}}$ s., 15/1 (1997), p. 17-23.

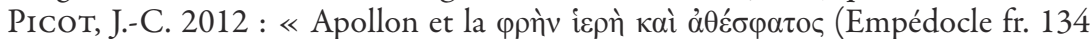
DK) », Anais de Filosofia Clássica, 6/11 (2012), p. 1-31.

Primavesi, O. 2013 : « Empedokles », dans H. Flashar, D. Bremer \& G. Rechenauer (éd.), Grundriss der Geschichte der Philosophie : Die Philosophie der Antike, 1/2, Frühgriechische Philosophie, Basel, 2013, p. 667-739.

Provencal, V. L. 2015 : Sophist Kings : Persians as Other in Herodotus, London - New Delhi-New York-Sidney, 2015 (Bloomsbury Classical Studies Monographs).

RaAflaub, K. A. 2000 : «Den Olympier herausfordern ? Prozesse im Umkreis des Perikles », dans L. Burckhardt \& J. von Ungern-Sternberg (éd.), Grosse Prozesse im Antiken Athen, München, 2000, p. 96-113.

Richards, H. 1902 : « Varia », The Classical Review, 16/8 (1902), p. 393-397.

Robbiano, C. 2006 : Becoming Being : On Parmenides' Transformative Philosophy, Sankt Augustin, 2006 (International Pre-Platonic Studies, 5).

Romeo, C. 1988 (éd.) : Demetrio Lacone, La poesia (PHerc. 188 e 1014), Napoli, 1988 (La Scuola di Epicuro, 9).

Rubel, A. 2000 : Stadt in Angst: Religion und Politik in Athen während des Peloponnesischen Krieges, Darmstadt, 2000 (Edition Universität).

SASsi, M. M. 1987 : « Platone, Fedone 108d: Glauco, Protagora, il mito », La Parola del Passato, 42 (1987), p. 27-34.

- 2011 : «Senofane fra i sofisti : dai limiti della conoscenza (21B34 DK) al paradosso eristico (Plat. Men. 80d5-e5) », Méthexis, 24 (2011), p. 7-20.

- 2013 : «Where Epistemology and Religion Meet : What Do(es) the God(s) Look Like ? », Rhizomata, 1/2 (2013), p. 283-307.

Schirren, T. \& T.Zinsmaier 2003 (éd.) : Die Sophisten : ausgewählte Texte : Griechisch/deutsch, Stuttgart, 2003 (Universal-Bibliothek, 18264).

Scholten, C. 2015 (éd.) : Theodoret, De Graecarum affectionum curatione = Heilung der griechischen Krankheiten, übers., eingel. und mit Anmerkungen versehen, Leiden-Boston, 2015 (Supplements to Vigiliae Christianae, 126).

Schöpsdau, K. 2011 (trad.) : Platon, Werke : Übersetzung und Kommentar, 9, 2, Nomoi (= Gesetze) : Buch VIII-XII, Göttingen, 2011.

Schorn, S. 2004 (éd.) : Satyros aus Kallatis, Sammlung der Fragmente mit Kommentar, Basel, 2004.

- 2012 : «Chamaeleon : Biography and Literature Peri tou deina », dans A. Martano, E. Matelli \& D. Mirhady (éd.), Praxiphanes of Mytilene and Chamaeleon of Heraclea, New Brunswick, 2012, p. 411-444 (Rutgers University Studies in Classical Humanities, 18). 
Sedley, D. 2013 : « The Atheist Underground », dans V. Harte \& M. Lane (éd.), Politeia in Greek and Roman Philosophy, Cambridge, 2013, p. 329-348.

Smith, M. F. 2003 (éd.) : Supplement to Diogenes of Oinoanda : The Epicurean Inscription, Napoli, 2003 (La Scuola di Epicuro. Supplemento, 3).

Solana Dueso, J. 2013 (trad.) : Los sofistas, Testimonios y fragmentos, traducción, introducción y notas, Madrid, 2013.

Susanetri, D. 1999 : «Foglie caduche e fragili genealogie », Prometheus, 25/2 (1999), p. 97-116.

Tordesillas, A. 2009 : « Platão, Protágoras e o homem-medida », Dissertatio, 29 (2009), p. 11-42 (DOI : http://dx.doi.org/10.15210/dissertatio.v29i0.8817).

Tulli, M. 2008 : «Esiodo e il sogno di Callimaco », Seminari Romani di Cultura Greca, 11/2 (2008), p. 185-198.

Untersteiner, M. 1961 (éd.) : Sofisti, Testimonianze e frammenti, I, Protagora e Seniade, Firenze, 1961 (Biblioteca di Studi Superiori).

- 1993 : Les Sophistes, traduit de l'italien et présenté par Alonso Tordesillas ; préface de Gilbert Romeyer-Dherbey, deuxième édition revue et notablement augmentée avec un appendice sur « Les origines sociales de la sophistique », Paris, 1993 (Bibliothèque d'histoire de la philosophie).

Van Riel, G. 2013 : Plato's Gods, Aldershot, 2013 (Ashgate Studies in the History of Philosophical Theology).

Versnel, H. S. 2011 : Coping With the Gods: Wayward Readings in Greek Theology, Leiden, 2011 (Religions in the Graeco-Roman World, 173).

Wallace, R. W. 1994 : « Private Lives and Public Enemies : Freedom of Thought in Classical Athens », dans A. L. Boegehold \& A. C. Scafuro (éd.), Athenian Identity and Civic Ideology, Baltimore, 1994, p. 127-155.

- 1996: «Book-Burning in Ancient Athens », dans R. W. Wallace \& E. M. Harris (éd.), Transitions to Empire : Studies in Greco-Roman History 360-146 B.C. in honor of E. Badian, Norman, 1996, p. 226-240 (Oklahoma Series in Classical Culture, 21).

West, M. L. 1972 (éd.) : Iambi et elegi Graeci ante Alexandrum cantati, II : Callinus, Mimnermus, Semonides, Solon, Tyrtaeus, Minora adespota, Oxford, 1972.

Whitmarsh, T. 2015 : Battling the Gods: Atheism in the Ancient World, New York, 2015.

Wilamowitz-Moellendorff, U.von 1911 : « Ein Stück aus dem Ancoratus des Epiphanios », Sitzungsberichte der königlich preussischen Akademie der Wissenschaften, 38/2 (1911), p. 759-772.

WiniarczyK, M. 1976 : « Der erste Atheistenkatalog des Kleitomachos », Philologus, 120 (1976), p. 32-46.

— 1984: «Wer galt im Altertum als Atheist ? », Philologus, 128 (1984), p. 157-183.

- 2016 : Diagoras of Melos: A Contribution to the History of Ancient Atheism, translated from Polish by Witold Zbirohowski-Koscia, Berlin-Boston, 2016 (Beiträge zur Altertumskunde, Bd 350).

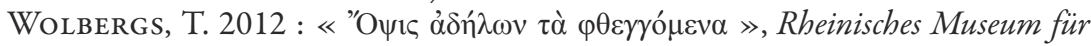
Philologie, 155/2 (2012), p. 113-127.

ZILIOLI, U. 2007 : Protagoras and the Challenge of Relativism: Plato's Subtlest Enemy, Aldershot, 2007 (Ashgate New Critical Thinking in Philosophy). 